CERN-TH.7334/94

IEM-FT-87/94

hep-ph/9407389

\title{
The lightest Higgs boson mass in the Minimal Supersymmetric Standard Model*
}

\author{
J.A. $\operatorname{Casas}^{1,2}$, J. R. Espinosa ${ }^{2 \dagger} \uparrow$ M. Quirós ${ }^{2}$ and A. Riotto ${ }^{2 \ddagger}$ \\ ${ }^{1}$ CERN, TH Division, CH-1211 Geneva 23, Switzerland \\ ${ }^{2}$ Instituto de Estructura de la Materia, CSIC \\ Serrano 123, 28006-Madrid, Spain
}

\begin{abstract}
We compute the upper bound on the mass of the lightest Higgs boson in the Minimal Supersymmetric Standard Model in a model-independent way, including leading (one-loop) and next-to-leading order (two-loop) radiative corrections. We find that (contrary to some recent claims) the two-loop corrections are negative with respect to the one-loop result and relatively small $(\lesssim 3 \%)$. After defining physical (pole) top quark mass $M_{t}$, by including QCD self-energies, and physical Higgs mass $M_{H}$, by including the electroweak self-energies $\Pi\left(M_{H}^{2}\right)-\Pi(0)$, we obtain the upper limit on $M_{H}$ as a function of supersymmetric parameters. We include as supersymmetric parameters the scale of supersymmetry breaking $M_{S}$, the value of $\tan \beta$ and the mixing between stops $X_{t}=A_{t}+\mu \cot \beta$ (which is responsible for the threshold correction on the Higgs quartic coupling). Our results do not depend on further details of the supersymmetric model. In particular, for $M_{S} \leq 1 \mathrm{TeV}$, maximal threshold effect $X_{t}^{2}=6 M_{S}^{2}$ and any value of $\tan \beta$, we find $M_{H} \leq 140 \mathrm{GeV}$ for $M_{t} \leq 190 \mathrm{GeV}$. In the particular scenario where the top is in its infrared fixed point we find $M_{H} \leq 86 \mathrm{GeV}$ for $M_{t}=170 \mathrm{GeV}$.
\end{abstract}

CERN-TH.7334/94

July 1994

*Work partly supported by CICYT under contract AEN94-0928, and by the European Union under contract No. CHRX-CT92-0004.

†Supported by a grant of Comunidad de Madrid, Spain.

${ }^{\ddagger}$ On leave of absence from International School of Advanced Studies, ISAS, Trieste. 


\section{Introduction}

There are good reasons to believe that the Standard Model (SM) is not the ultimate theory since it is unable to answer many fundamental questions. One of them, why and how the electroweak and the Planck scales are so hierarchically separated, has motivated the proposal of the Minimal Supersymmetric extension of the Standard Model (MSSM) as the underlying theory at scales of order $1 \mathrm{TeV}$. Indeed, the supersymmetric scale cannot be very large not to spoil the solution to the hierarchy problem, but the ambiguity about its maximal allowable size makes it very difficult to give modelindependent predictions testable in accelerators.

Fortunately, the supersymmetric predictions for the upper bound on the lightest Higgs boson mass represent a (perhaps unique) exception to this rule. Therefore, they are crucial for the experimental verification of supersymmetry. This importance is reinforced by the fact that uncovering the Higgs boson of the SM is one of the main challenges for present (LEP, Tevatron) and future (LEP-200, LHC) accelerators. Much work has been recently devoted to this subject [1-10], but still there is a substantial disagreement on the final results (see e.g. $[5,9,10]$ ), especially concerning the two-loop corrections (which, as we will see, are crucial).

The aim of this paper is to evaluate the two-loop predictions of the MSSM on the lightest Higgs mass in a consistent and model-independent way. In fact, our results will not depend on the details of the MSSM (e.g. the assumption or not of universality of the soft breaking terms, gauge and Yukawa unification, etc.). Actually, when we will refer to the MSSM, we will simply mean the supersymmetric version of the SM with minimal particle content. The consistency of the approach will allow us to show up the reasons of the disagreement between previous results. We also give the predictions on the Higgs mass for a particularly appealing scenario, namely the assumption that the recently detected top quark [11] has the Yukawa coupling in its infrared fixed point. It will turn out that in this case the Higgs boson should be just around the corner.

Let us briefly review the status of the subject and the most recent contributions on it. The MSSM has an extended Higgs sector with two Higgs doublets with opposite hypercharges: $H_{1}$, responsible for the mass of the charged leptons and the down-type quarks, and $H_{2}$, which gives a mass to the up-type quarks. After the Higgs mechanism there remain three physical scalars, two CP-even and one CP-odd Higgs bosons. In particular, the lightest CP-even Higgs boson mass satisfies the tree-level bound

$$
m_{H}^{2} \leq M_{Z}^{2} \cos ^{2} 2 \beta,
$$

where $\tan \beta=v_{2} / v_{1}$ is the ratio of the Vacuum Expectation Values (VEV's) of the neutral components of the two Higgs fields $H_{2}$ and $H_{1}$, respectively. The relation (1.1) implies that $m_{H}^{2}<M_{Z}^{2}$, for any value of $\tan \beta$ which, in turn, implies that it should be found at LEP-200 [1]. However, the tree level relation (1.1) is spoiled by one-loop radiative corrections, which were computed by several groups using: the effective potential approach [2], diagrammatic methods [3] and renormalization group 
(RG) techniques [4]. All methods found excellent agreement with each other and large radiative corrections, mainly controlled by the top Yukawa coupling, which could make the lightest CP-even Higgs boson to escape experimental detection at LEP-200. In particular, the RG approach (which will be followed in the present paper) is based on the fact that supersymmetry decouples and below the scale of supersymmetry breaking $M_{S}$ the effective theory is the SM. Assuming $M_{Z}^{2} \ll M_{S}^{2}$ the tree-level bound (1.1) is saturated at the scale $M_{S}$ and the effective SM at scales between $M_{Z}$ and $M_{S}$ contains the Higgs doublet

$$
H=H_{1} \cos \beta+i \sigma_{2} H_{2}^{*} \sin \beta,
$$

with a quartic coupling taking, at the scale $M_{S}$, the (tree level) value

$$
\lambda=\frac{1}{4}\left(g^{2}+g^{\prime 2}\right) \cos ^{2} 2 \beta .
$$

In these analyses [i] the Higgs mass was considered at the tree-level, improved by oneloop renormalization group equations (RGE) in the $\gamma$-and $\beta$-functions, thus collecting all leading logarithm corrections.

Since the relative size of one-loop corrections to the Higgs mass is large (mainly for large top quark mass and/or small tree level Higgs mass) it was compelling to analyze them at the two-loop level. A first step in that direction was given in ref. [5] where two-loop RGE improved tree level Higgs masses were considered. It was found that two-loop corrections were negative and small. In ref. [5] the Higgs mass received all leading logarithm and part of the next-to-leading logarithm corrections. Subsequent studies of the effective potential improved by the RGE [6, 7, 8] have shown that the L-loop improved effective potential with ( $\mathrm{L}+1)$-loop RGE is exact up to Lth-to-leading logarithm order $[7]$. This means that for fully taking into account all next-to-leading logarithm corrections the one-loop effective potential (improved by two-loop RGE) is needed.

Finally, two papers [9, 10] have recently appeared aiming to refine the two-loop analysis of ref. [5]. The authors of ref. [9], following the RG approach, find that the two-loop correction to the Higgs mass is positive and sizeable, whereas the authors of ref. [10], following diagrammatic [3] and effective potential [2] approaches in the framework of the MSSM with various approximations, find that the two-loop correction to the Higgs mass is negative and sizeable in contradiction with ref. [9]. As we will see in detail in section 5 we are in disagreement with the results of ref. [9] (showing how a correct treatment of all the relevant effects would make the results presented in ref. [9] to agree with ours), and in agreement with the overall result of ref. [10] (though not with the relative size of the two-loop corrections).

In this paper we use the RG approach and the SM one-loop improved effective potential with two-loop RGE to compute the lightest Higgs mass of the MSSM up to next-to-leading logarithm order. At this level of approximation, one has to keep control

\footnotetext{
${ }^{1}$ Strictly speaking this has been proven [7] for a theory with a single mass scale.
} 
on the validity of the perturbative expansion for $V$. In particular, although the whole effective potential is scale-invariant, the one-loop approximation is not, thus one has to be careful about the choice of the renormalization scale. As we will see this fact is at the origin of some misunderstandings in previous works. Furthermore, since the Higgs mass is computed to one-loop order there are a number of one-loop effects that need to be considered for the consistency of the procedure:

i) As it is explained in Appendix A, the tree level quartic coupling (1.3) receives one-loop threshold contributions at the $M_{S}$ scale. These are given by

$$
\Delta \lambda=\frac{3 h_{t}^{4}}{16 \pi^{2}} \frac{X_{t}^{2}}{M_{S}^{2}}\left(2-\frac{X_{t}^{2}}{6 M_{S}^{2}}\right),
$$

where $h_{t}$ is the top Yukawa coupling in the SM and

$$
X_{t}=A_{t}+\mu \cot \beta
$$

is the stop mixing.

The correction (1.4) has a maximum for $X_{t}^{2}=6 M_{S}^{2}$. For that reason, in our numerical applications we will take two cases: $X_{t}=0$, i.e. no mixing, and $X_{t}^{2}=6 M_{S}^{2}$, i.e. maximal threshold effect. Notice also that $X_{t}^{2}=6 M_{S}^{2}$ is barely consistent with the bound from color conserving minimum [13], so the case of maximal threshold really represent a particularly extreme situation.

In addition to the previous effect, there appear effective higher order operators ( $D \geq 6$ ), which for $M_{S} \geq 1 \mathrm{TeV}$ turn out to be negligible (for details see Appendix A).

ii) One-loop contributions to the top quark self-energy relating the running top mass $m_{t}$ to the (physical) propagator pole top mass $M_{t}$. We will find this effect gives a negative and sizeable contribution to the Higgs mass

iii) One-loop contributions to the Higgs self-energy relating also the running Higgs mass $m_{H}$ to the physical Higgs mass $M_{H}$ (for details see Appendix B). We will find scale dependent contributions to the self-energy, removing the scale dependence of $M_{H}$, and other scale independent contributions. This effect will be found to be positive in all cases, partially cancelling the previous effect.

The contents of this paper are as follows: In section 2 we present and analyze the one-loop effective potential, for the effective SM in the range between $M_{Z}$ and $M_{S}$, improved by the RGE. We compare different treatments of the effective potential and define the running Higgs masses. Our treatment of the effective potential is presented in detail in section 3 , and the numerical results are presented in section 4 , where we make use of effects (i)-(iii) above. Numerical and conceptual comparison with other recent approaches is presented in section 5, and our conclusions are drawn in section 6. In Appendix B we present analytic expressions for the one-loop contribution to the Higgs boson self-energy $\Pi\left(q^{2}\right)-\Pi(0)$, computed in the 't Hooft-Landau gauge and using the $\overline{M S}$ renormalization scheme. We also comment on the introduction of the Higgs sector both in the effective potential and in the Higgs boson self-energy. 


\section{The one-loop effective potential}

Our starting point in this section will be the effective potential of the Standard Model. This can be written in the 't Hooft-Landau gauge as [8]

$$
V_{e f f}\left(\mu(t), \lambda_{i}(t) ; \phi(t)\right) \equiv V_{0}+V_{1}+\cdots,
$$

where $\lambda_{i} \equiv\left(g, g^{\prime}, \lambda, h_{t}, m^{2}\right)$ runs over all dimensionless and dimensionful couplings and $V_{0}, V_{1}$ are respectively the tree level potential and the one-loop correction, namely

$$
\begin{gathered}
V_{0}=-\frac{1}{2} m^{2}(t) \phi^{2}(t)+\frac{1}{8} \lambda(t) \phi^{4}(t) \\
V_{1}=\frac{1}{64 \pi^{2}}\left\{6 m_{W}^{4}(t)\left[\log \frac{m_{W}^{2}(t)}{\mu^{2}(t)}-\frac{5}{6}\right]+3 m_{Z}^{4}(t)\left[\log \frac{m_{Z}^{2}(t)}{\mu^{2}(t)}-\frac{5}{6}\right]\right. \\
\left.-12 m_{t}^{4}(t)\left[\log \frac{m_{t}^{2}(t)}{\mu^{2}(t)}-\frac{3}{2}\right]\right\},
\end{gathered}
$$

where we have used the $\overline{M S}$ renormalization scheme [12]. The parameters $\lambda(t)$ and $m(t)$ are the Standard Model quartic coupling and mass, running with the RGE, while the running Higgs field is

$$
\phi(t)=\xi(t) \phi_{c}
$$

$\phi_{c}$ being the classical field and

$$
\xi(t)=e^{-\int_{0}^{t} \gamma\left(t^{\prime}\right) d t^{\prime}}
$$

where $\gamma(t)$ is the Higgs field anomalous dimension. $V_{1}$ in eq.(2.3) contains the radiative corrections where only the top quark and the $W, Z$ gauge bosons are propagating in the loop. For the moment we will disregard those coming from the Higgs and Goldstone boson propagation [2]. They can be easily introduced, as shown in Appendix B, without altering the numerical results that are obtained in this paper. The mass parameters in (2.3) are given by

$$
\begin{aligned}
m_{W}^{2}(t) & =\frac{1}{4} g^{2}(t) \phi^{2}(t) \\
m_{Z}^{2}(t) & =\frac{1}{4}\left[g^{2}(t)+g^{\prime 2}(t)\right] \phi^{2}(t), \\
m_{t}^{2}(t) & =\frac{1}{2} h_{t}^{2}(t) \phi^{2}(t),
\end{aligned}
$$

\footnotetext{
${ }^{2}$ In particular, the Goldstone boson contribution to the effective potential generates, on the running Higgs mass, an infrared logarithmic divergence. This divergence is cancelled when the physical Higgs mass is considered, as will be shown in Appendix B.
} 
where $g, g^{\prime}$ and $h_{t}$ are the $S U(2), U(1)$ and top Yukawa coupling, respectively. Finally the scale $\mu(t)$ is related to the running parameter $t$ by

$$
\mu(t)=\mu e^{t}
$$

where $\mu$ is a fixed scale, that we will take equal to the physical $Z$ mass, $M_{Z}$.

The complete effective potential $V_{\text {eff }}\left(\mu(t), \lambda_{i}(t) ; \phi(t)\right)$ and its $n-t h$ derivative are scale-independent (see e.g. ref. [B] ), i.e.

$$
\frac{d V_{e f f}^{(n)}}{d t}=0
$$

where

$$
V_{e f f}^{(n)} \equiv \xi^{n}(t) \frac{\partial^{n}}{\partial \phi(t)^{n}} V_{e f f}\left(\mu(t), \lambda_{i}(t) ; \phi(t)\right)
$$

The above property allows in principle to fix a different scale for each value of the classical field $\phi_{c}$, i.e. $\mu(t)=f\left(\phi_{c}\right)$ or, equivalently, $t=t\left(\phi_{c}\right)=\log \left(f\left(\phi_{c}\right) / \mu\right)$. In that case $V_{\text {eff }}$ becomes $V_{\text {eff }}\left(\phi_{c}\right) \equiv V_{\text {eff }}\left(f\left(\phi_{c}\right), \lambda_{i}\left(t\left(\phi_{c}\right)\right)\right.$. Then, from (2.8) and (2.9) one can readily prove that

$$
\left.V_{e f f}^{(n)}\right|_{t=t\left(\phi_{c}\right)}=\frac{d^{n} V_{e f f}\left(\phi_{c}\right)}{d \phi_{c}^{n}},
$$

as expected. This procedure has been used in some previous works?. However, one should notice that even though the whole effective potential is scale invariant, the oneloop approximation is not. Therefore one would need a criterium to fix the function $f\left(\phi_{c}\right)$ : the only possible one would be to minimize the radiative corrections improving, so, perturbative expansion. Assuming that only one field, say $f$ with squared mass $m_{f}^{2}(t)$, is contributing to the one-loop radiative corrections, or that the latter are dominated by this field, the most natural choice would be $\mu^{2}(t)=m_{f}^{2}(t)$. Nevertheless this approach has (in our context) some drawbacks:

i) The $t$-dependence of $m_{f}^{2}(t)$ is implicit through the RGE and so the function $t=t\left(\phi_{c}\right)$ cannot be explicitly written.

ii) In most cases, in particular in our one-loop correction (2.3), one cannot assume, within the required degree of accuracy, that loop corrections are dominated by only one field.

iii) When computing $V_{e f f}^{(n)}\left(\phi_{c}\right)$ in 2.10 the vacuum energy $\Omega\left(\lambda_{i}(t), \mu(t)\right)$ has to be specified since it also acquires a $\phi_{c}$-dependence through the variable change $t=t\left(\phi_{c}\right)$. Furthermore, the whole dependence on $\phi_{c}$ becomes now much more involved.

\footnotetext{
${ }^{3}$ E.g. in studies of the effective potential stability [8] to control large logarithms that can arise for large field values. It has also been used in ref. [9], but as we will see, it is an unnatural complication in this context.
} 
iv) The last, but not the least, drawback is that once one has chosen a particular function $t\left(\phi_{c}\right)$, one looses track of scale invariance and so there is no way of checking how good the approximation is at the minimum of the effective potential.

For the above mentioned reasons we will keep $t$ and $\phi_{c}$ as independent variables. We will minimize the effective potential (2.1), truncated at one-loop, at some fixed scale $t=t^{*}$, i.e.

$$
\left.\frac{\partial V_{\text {eff }}}{\partial \phi\left(t^{*}\right)}\right|_{\phi\left(t^{*}\right)=\left\langle\phi\left(t^{*}\right)\right\rangle}=0,
$$

which will determine the VEV $\left\langle\phi\left(t^{*}\right)\right\rangle$. Our criterium to fix the scale $t^{*}$ will be explained in the next section. The scale independence of the whole effective potential implies that

$$
\frac{d}{d t} \frac{\langle\phi(t)\rangle}{\xi(t)}=0 .
$$

Therefore, assuming that $t^{*}$ lies in the region where the one-loop approximation to the effective potential is reliable, the VEV of the field at any scale can be obtained throught

$$
\langle\phi(t)\rangle=\left\langle\phi\left(t^{*}\right)\right\rangle \frac{\xi(t)}{\xi\left(t^{*}\right)} .
$$

Accordingly, we must impose

$$
v=\left\langle\phi\left(t_{Z}\right)\right\rangle=\left\langle\phi\left(t^{*}\right)\right\rangle \frac{\xi\left(t_{Z}\right)}{\xi\left(t^{*}\right)},
$$

where $t_{Z}$ is defined as $\mu\left(t_{Z}\right)=M_{Z}$ and

$$
v=\left(\sqrt{2} G_{\mu}\right)^{-1 / 2}=246.22 \mathrm{GeV},
$$

is the "measured" VEV for the Higgs field [16].

We can trade $\left\langle\phi\left(t^{*}\right)\right\rangle$ by $m^{2}\left(t^{*}\right)$ from the condition of minimum (2.11), which translates, using (2.14), into the boundary condition for $m^{2}(t)$ :

$$
\begin{gathered}
\frac{m^{2}\left(t^{*}\right)}{v^{2}}=\frac{1}{2} \lambda\left(t^{*}\right) \xi^{2}\left(t^{*}\right)+\frac{3}{64 \pi^{2}} \xi^{2}\left(t^{*}\right)\left\{\frac{1}{2} g^{4}\left(t^{*}\right)\left[\log \frac{g^{2}\left(t^{*}\right) \xi^{2}\left(t^{*}\right) v^{2}}{4 \mu^{2}\left(t^{*}\right)}-\frac{1}{3}\right]\right. \\
+\frac{1}{4}\left[g^{2}\left(t^{*}\right)+g^{\prime 2}\left(t^{*}\right)\right]^{2}\left[\log \frac{\left[g^{2}\left(t^{*}\right)+g^{\prime 2}\left(t^{*}\right)\right] \xi^{2}\left(t^{*}\right) v^{2}}{4 \mu^{2}\left(t^{*}\right)}-\frac{1}{3}\right] \\
\left.-4 h_{t}^{4}\left(t^{*}\right)\left[\log \frac{h_{t}^{2}\left(t^{*}\right) \xi^{2}\left(t^{*}\right) v^{2}}{2 \mu^{2}\left(t^{*}\right)}-1\right]\right\} .
\end{gathered}
$$

\footnotetext{
${ }^{4}$ Notice that $\langle\phi(t)\rangle$ defined by (2.13) coincides with the value of $\phi(t)$ which minimizes the whole effective potential. In the one-loop approximation that we are using this is no longer true, as we will see later on.

${ }^{5} \mathrm{We}$ are neglecting here one-loop electroweak radiative corrections to the muon $\beta$-decay slightly modifying the relation (2.15), see e.g. ref. [15]. We thank S. Peris for a discussion on this point.
} 
We can define now $m_{H}\left(t^{*}\right)$ as the second derivative of the effective potential at the minimum, evaluated at the scale $t^{*}$, i.e.

$$
\begin{aligned}
m_{H}^{2}\left(t^{*}\right) & =\left.\frac{\partial^{2} V_{\text {eff }}}{\partial \phi\left(t^{*}\right)^{2}}\right|_{\phi\left(t^{*}\right)=\left\langle\phi\left(t^{*}\right)\right\rangle} \\
& =\lambda\left(t^{*}\right) \xi^{2}\left(t^{*}\right) v^{2}+\frac{3}{64 \pi^{2}} \xi^{2}\left(t^{*}\right) v^{2}\left\{g^{4}\left(t^{*}\right)\left[\log \frac{g^{2}\left(t^{*}\right) \xi^{2}\left(t^{*}\right) v^{2}}{4 \mu^{2}\left(t^{*}\right)}+\frac{2}{3}\right]\right. \\
& +\frac{1}{2}\left[g^{2}\left(t^{*}\right)+g^{\prime 2}\left(t^{*}\right)\right]^{2}\left[\log \frac{\left[g^{2}\left(t^{*}\right)+g^{\prime 2}\left(t^{*}\right)\right] \xi^{2}\left(t^{*}\right) v^{2}}{4 \mu^{2}\left(t^{*}\right)}+\frac{2}{3}\right] \\
& \left.-8 h_{t}^{4}\left(t^{*}\right) \log \frac{h_{t}^{2}\left(t^{*}\right) \xi^{2}\left(t^{*}\right) v^{2}}{2 \mu^{2}\left(t^{*}\right)}\right\},
\end{aligned}
$$

where we have used (2.16). At an arbitrary scale $t$ we can use the scale independence property of the whole potential, see (2.9), and write

$$
m_{H}^{2}(t)=m_{H}^{2}\left(t^{*}\right) \frac{\xi^{2}\left(t^{*}\right)}{\xi^{2}(t)},
$$

which will be our definition of running mass.

In the region where our approximated effective potential is scale invariant, definition (2.18) should be equivalent to taking the second derivative with respect to $\phi(t)$, evaluate it at $\langle\phi(t)\rangle$ and use, for the $\mathrm{VEV}$, the relation (2.13), i.e.

$$
\begin{aligned}
m_{H, d e r}^{2}(t) & =\left.\frac{\partial^{2} V_{\text {eff }}}{\partial \phi(t)^{2}}\right|_{\phi(t)=\langle\phi(t)\rangle} \\
& =-m^{2}(t)+\frac{3}{2} \xi^{2}(t) v^{2}\left\{\lambda(t)+\frac{1}{8 \pi^{2}}\left\{\frac{1}{8} g^{4}(t)\left[3 \log \frac{g^{2}(t) \xi^{2}(t) v^{2}}{4 \mu^{2}(t)}+1\right]\right.\right. \\
& +\frac{1}{16}\left[g^{2}(t)+g^{\prime 2}(t)\right]^{2}\left[3 \log \frac{\left[g^{2}(t)+g^{\prime 2}(t)\right] \xi^{2}(t) v^{2}}{4 \mu^{2}(t)}+1\right] \\
& \left.\left.-h_{t}^{4}(t)\left[3 \log \frac{h_{t}^{2}(t) \xi^{2}(t) v^{2}}{2 \mu^{2}(t)}-1\right]\right\}\right\} .
\end{aligned}
$$

Of course, exact scale invariance would imply that (2.18) and (2.19) are equal. This will allow to cross check the reliability of our approach.

\section{Our approach}

In this section we will describe our approach to the problem of determination of the Higgs mass (2.18). The effective potential is written in (2.2) and (2.3), and the parameters on which it depends, $\lambda(t), g(t), g^{\prime}(t), g_{3}(t), h_{t}(t), \xi(t)$ and $m^{2}(t)$, satisfy a system of coupled RGE with $t$-dependence governed by $\beta_{\lambda}, \beta_{g}, \beta_{g^{\prime}}, \beta_{g_{3}}, \beta_{h_{t}}, \gamma, \beta_{m^{2}}$, i.e. the corresponding $\beta$-functions and anomalous dimension of the Higgs field, which

are evaluated to one- or two-loop order [8]. In fact, we will often consider two cases: 
a) The one-loop case, where $\beta$ and $\gamma$-functions are considered to one-loop and the effective potential is approximated by the tree level term (2.2). In this case the leading logarithms are resummed to all-loop in the effective potential. The Higgs mass includes then all-loop leading logarithm contributions [6, [1].

b) The two-loop case, where $\beta$ and $\gamma$-functions in the RGE are considered to two-loop order and the effective potential is considered in the one-loop approximation, (2.2) and (2.3). In this case the leading and next-to-leading logarithms are resummed to all-loop in the effective potential. The Higgs mass includes then all-loop leading and next-to-leading logarithm contributions [7].

We will impose as boundary conditions:

- For the gauge couplings:

$$
\begin{gathered}
g\left(M_{Z}\right)=0.650 \\
g^{\prime}\left(M_{Z}\right)=0.355 \\
g_{3}\left(M_{Z}\right)=1.23 .
\end{gathered}
$$

- For the top Yukawa coupling:

$$
h_{t}\left(m_{t}\right)=\frac{\sqrt{2} m_{t}}{v}
$$

where $m_{t}$ is the running mass: $m_{t}\left(\mu(t)=m_{t}\right)=m_{t}$.

- For the quartic coupling:

$$
\lambda\left(M_{S}\right)=\frac{1}{4}\left[g^{2}\left(M_{S}\right)+g^{\prime 2}\left(M_{S}\right)\right] \cos ^{2} 2 \beta+\frac{3 h_{t}^{4}\left(M_{S}\right)}{16 \pi^{2}}\left(2 \frac{X_{t}^{2}}{M_{S}^{2}}-\frac{X_{t}^{4}}{6 M_{S}^{4}}\right) .
$$

- For the mass $m^{2}(t)$ we will take as boundary condition the value determined by eq. (2.16) at the scale $t^{*}$.

Note that the previous boundary conditions depend on the values of $m_{t}, t^{*}$ (i.e. the minimization scale) and the supersymmetric parameters $M_{S}, X_{t}$ and $\tan \beta$.

Our main task now will be to determine the optimal minimization scale $t^{*}$ as a function of $m_{t}$. Our criterion will be that $t^{*}$ be in the region where the effective potential is more scale independent. We estimate this in the following way. For fixed $t^{*}$ and $m_{t}$, all boundary conditions (3.1)-(3.3) are determined and the effective potential (2.1) can be computed for any value of $t$. Thus the corresponding value of $\phi(t)$, say $\phi_{\min }(t)$, that minimizes the one-loop potential can be numerically evaluated. Had we considered the whole effective potential, its scale independence property (2.12) would 
imply that $\phi_{\min }(t) / \xi(t)=\langle\phi(t)\rangle / \xi(t)$, where $\langle\phi(t)\rangle$ has been defined in (2.13), is $t$ independent. Since the one-loop approximation is not exactly scale independent the most appropriate scale is the scale $t_{s}$ for which $\phi_{\min }(t) / \xi(t)$ has a stationary point, i.e.

$$
\left.\frac{d}{d t} \frac{\phi_{\min }(t)}{\xi(t)}\right|_{t=t_{s}}=0 .
$$

Of course the scale at which (3.4) occurs depends on $t^{*}$ and $m_{t}$, i.e. $t_{s} \equiv t_{s}\left(t^{*}, m_{t}\right)$. Therefore, for a given value of $m_{t}$ the optimal scale $t^{*}$ is defined as

$$
t_{s} \equiv t_{s}\left(t^{*}, m_{t}\right)=t^{*}
$$

i.e. it is the scale which simultaneously defines the boundary condition (2.16) and agrees with the extremal of the function $\phi_{\min }(t) / \xi(t)$.

This procedure is illustrated in Fig. 1, where we plot $\phi_{\min }(t) / \xi(t)$ vs. $\mu(t)$ for $m_{t}=160 \mathrm{GeV}$ and supersymmetric parameters $M_{S}=1 \mathrm{TeV}, X_{t}=0, \cos ^{2} 2 \beta=1$. The solid curve shows the two-loop result whose stationary point satisfies eq. (3.5) and therefore defines the optimal scale $t^{*}$. Note that there is no fine tuning in the choice (3.5) since any point near the stationary point would be equally appropriate because the curve $\phi_{\min }(t) / \xi(t)$ is very flat in that region. We have also shown in Fig. 1 the curve $\phi_{\min }(t) / \xi(t)$ in the one-loop approximation (dashed curve) with $\mu\left(t^{*}\right)$ fixed by the two-loop result. We can see that the one-loop curve is much steeper than the two-loop curve, which means that the one-loop result, i.e. the tree level potential (2.2) improved by the one-loop RGE, is far from being scale independent at any scale. This feature has also been observed for the MSSM one-loop effective potential [17.

We plot in Figs. 2a,b,c,d, $\mu\left(t^{*}\right)$ as a function of $m_{t}$ for the different values of supersymmetric parameters $M_{S}=1,10 \mathrm{TeV} ; X_{t}^{2}=0,6 M_{S}^{2}$. In all plots the solid curve corresponds to $\cos ^{2} 2 \beta=1$ and the dashed curve to $\cos ^{2} 2 \beta=0$.

Once we have determined $\mu\left(t^{*}\right)$ for fixed values of $m_{t}$ and all supersymmetric parameters, the Higgs running mass $m_{H}(t)$ is given by (2.18), while the mass defined as the second derivative of the effective potential $m_{H, d e r}(t)$ is given by (2.19). By definition both masses coincide at $t^{*}$

$$
m_{H}\left(t^{*}\right)=m_{H, d e r}\left(t^{*}\right),
$$

and the ratio $m_{H, d e r}(t) / m_{H}(t)$ should be equal to one for an exactly scale independent effective potential. Consistency of our procedure requires the curve $m_{H, d e r}(t) / m_{H}(t)$ to be flat in the region where we minimize, i.e. at $t^{*}$. In Fig. 3 we plot $m_{H, d e r}(t) / m_{H}(t)$ for $m_{t}=160 \mathrm{GeV}$ and the values of supersymmetric parameters as in Fig. $1, M_{S}=1$ $\mathrm{TeV}, X_{t}=0, \cos ^{2} 2 \beta=1$. We see that at the point $\mu\left(t^{*}\right)$ the curve is in its flat region, though $\mu\left(t^{*}\right)$ does not exactly coincide with the extremum of $m_{H, d e r}(t) / m_{H}(t)$.

Neither $m_{H}$ nor $m_{t}$ are physical masses. They are computed from the effective potential, i.e. at zero external momentum, and need to be corrected by the corresponding polarizations to obtain the propagator pole physical masses. This will be done in section 4. For the time being, and just to compare with other results in the literature, we 
will neglect the shift to the physical poles and define the top mass by the boundary condition (3.2) and the Higgs mass by the usual condition

$$
m_{H}\left(\mu(t)=m_{H}\right)=m_{H} .
$$

In this way the Higgs mass $m_{H}$ can be unambiguously determined. We plot in Fig. 4 $m_{H}$ as a function of $m_{t}$ for the values of supersymmetric parameters $M_{S}=1 \mathrm{TeV}$, $X_{t}=0, \cos ^{2} 2 \beta=1$. The thin solid line corresponds to the two-loop result and the dashed-line corresponds to the one-loop result. (We will disregard for the moment the thick solid line, which corresponds to shifting the running masses to propagator poles.) The main feature that arises from Fig. 4 is that the two-loop corrections are negative with respect to the one-loop result. This feature is in qualitative agreement with our previous two-loop result [5] (where the one-loop corrections to the effective potential where not considered) and with others from different authors [10]. This comparison will be done in some detail in section 5 .

\section{Numerical Results}

We will present, in this section, the numerical results on the Higgs mass, evaluated in the next-to-leading logarithm approximation, as a function of the top quark mass. The running top quark mass $m_{t}$ that we have been using in the previous section was evaluated at the scale $\mu(t)=m_{t}$, i.e. $m_{t}\left(m_{t}\right)=m_{t}$ (see eq.(3.2)). However the running mass does not coincide with the gauge invariant pole of the top quark propagator $M_{t}$. In the Landau gauge the relationship between the running $m_{t}$ and the physical (pole) mass $M_{t}$ is given by [14]

$$
M_{t}=\left[1+\frac{4}{3} \frac{\alpha_{s}\left(M_{t}\right)}{\pi}\right] m_{t}\left(M_{t}\right) .
$$

On the other hand, the running Higgs mass, $m_{H}(t)$, given by eq. (2.18), has a scale variation

$$
\frac{d m_{H}^{2}(t)}{d t}=2 \gamma m_{H}^{2}(t) .
$$

The propagator pole $M_{H}$ is related to the running mass through (see Appendix B)

$$
M_{H}^{2}=m_{H}^{2}(t)+\operatorname{Re} \Pi\left(M_{H}^{2}\right)-\operatorname{Re} \Pi(0),
$$

where $\Pi\left(q^{2}\right)$ is the (renormalized) self-energy of the Higgs boson. In our calculation (case (b) in section 3) it is enough to compute the Higgs self-energies at the one-loop level since we are computing the Higgs masses to one-loop. The imaginary part of $\Pi\left(M_{H}^{2}\right)-\Pi(0)$ contributes to the Higgs width. Assuming $M_{H}<2 M_{W}$, the Higgs is stable at tree level, and eq. (4.3) reads

$$
M_{H}^{2}=m_{H}^{2}+\Pi\left(M_{H}^{2}\right)-\Pi(0) .
$$


The calculation of $\Pi\left(M_{H}^{2}\right)-\Pi(0)$ is presented in Appendix B. We note that the scale dependence (at the one-loop level) of $m_{H}(t)$, as provided by (4.2), is cancelled by the scale dependence of $\Pi\left(M_{H}^{2}\right)-\Pi(0)$. The only remaining scale dependence of $M_{H}$ comes from two-loop contributions. This feature is exhibited in Fig. 5 where we plot $M_{H}$ in the range of $\mu(t)$ between $M_{S}$ and $M_{Z}$ for $m_{t}=160 \mathrm{GeV}$ and the supersymmetric parameters $M_{S}=1 \mathrm{TeV}, X_{t}=0, \cos ^{2} 2 \beta=1$. We can see that $M_{H}$ has a negligible variation $(\sim 0.5 \mathrm{GeV})$ between $M_{S}$ and $M_{Z}$. For the sake of comparison we have also plotted the running mass $m_{H}(t)$ whose variation is more appreciable $(\sim 2.5 \mathrm{GeV})$. This effect is more accentuated for larger top masses.

We can see from (4.1) and from Fig. 4 that the effect of considering the pole mass $M_{t}$ is negative on the two-loop corrections to the Higgs mass, while the effect of $\Pi\left(M_{H}^{2}\right)-$ $\Pi(0)$ is positive $\left(\sim 2 \mathrm{GeV}\right.$ for $m_{t}=160 \mathrm{GeV}$ and $\sim 5 \mathrm{GeV}$ for $\left.m_{t}=215 \mathrm{GeV}\right)$ thus partially compensating the effect of (4.1). The global effect is negative and small as can be seen in Fig. 4. The thick solid line indicates $M_{H}$ as a function of $M_{t}$. It is below the thin solid line which was the two-loop evaluation of $m_{H}$ as a function of $m_{t}$. The comparison with the one-loop result (dashed line) shows that two-loop corrections are negative with respect to the one-loop result. Numerically they are small $(\sim 1 \mathrm{GeV})$ for $M_{t}=120 \mathrm{GeV}$ and larger $(\sim 6-7 \mathrm{GeV})$ for $M_{t}=220 \mathrm{GeV}$.

In Fig. 6a,b,c,d we plot $M_{H}$ as a function of $M_{t}$ for values of supersymmetric parameters $M_{S}=1,10 \mathrm{TeV} ; X_{t}^{2}=0,6 M_{S}^{2} ; \cos ^{2} 2 \beta=0,1$. In all cases the solid curve corresponds to $\cos ^{2} 2 \beta=1$ and the dashed curve to $\cos ^{2} 2 \beta=0$. Notice that the dependence on the mixing parameter $X_{t}$ is sizeable. In all the figures we have used the lower limit on the top quark mass, $M_{t}>120 \mathrm{GeV}$ at $95 \%$ C.L., from the CDF dilepton channel [11]. If we use the recent evidence for the top quark production at CDF with a mass $M_{t}=174 \pm 10_{-12}^{+13} \mathrm{GeV}$ [11] and the bounds for supersymmetric parameters $M_{S} \leq 1 \mathrm{TeV}$ and maximal threshold correction $X_{t}^{2}=6 M_{S}^{2}$, we obtain the absolute upper bound $M_{H}<140 \mathrm{GeV}$.

The dependence of $M_{H}$ on $\tan \beta$ is exhibited in Fig. 7 where we fix $M_{t}=170 \mathrm{GeV}$ and $M_{S}=1 \mathrm{TeV}$. The solid curve corresponds to the absolute upper bound for the mixing $X_{t}^{2}=6 M_{S}^{2}$ and the dashed curve to the case of zero mixing. Concerning the dependence of $M_{H}$ on the stop mixing $X_{t}$ parameter (or equivalently the stop splitting), we have found it to be sizeable, as can be seen from Figs. 6 .

\section{Connection with other approaches}

Some papers have recently appeared aiming to estimate the mass of the lightest Higgs boson up to next-to-leading order in the MSSM, and with apparently contradictory results. In this section we will comment on those papers in the context of our formalism and will exhibit the origin of the disagreements.

In ref. [9] the RG approach was used to estimate the mass of the lightest Higgs boson up to next-to-leading order. The relevant points of their calculation are the following: i) They considered the one-loop correction to the effective potential in the approximation 
$g=g^{\prime}=0$, ii) They neglected the wave-function renormalization leading to physical masses for the top quark and Higgs boson, iii) They minimized the effective potential at the scale $\mu\left(t^{*}\right)=v$. In addition they only consider the case of zero stop mixing, $X_{t}=0$.

Contrary to our results, they found that the two-loop correction is positive and sizeable with respect to the one-loop result. For instance in the typical case $M_{S}=1$ $\mathrm{TeV}, X_{t}=0, \cos ^{2} 2 \beta=1$, they find for $m_{t}=170 \mathrm{GeV}$ the two-loop result $m_{H} \sim 117$ $\mathrm{GeV}$, while we find for $M_{t}=170 \mathrm{GeV}$ the two-loop result $M_{H} \sim 111 \mathrm{GeV}$. We have been able to trace back the difference between the two results to the points i)-iii) above. In particular, the authors of ref. [9] find for the same values of the parameters a positive two-loop correction with respect to the one-loop result $\sim 7 \mathrm{GeV}$ while our two-loop result is smaller than the one-loop result by $\sim 3 \mathrm{GeV}$. To understand the origin of this discrepancy we have plotted in Fig. 8 the physical Higgs mass $M_{H}$ as a function of the minimization scale $\mu\left(t^{*}\right)$ for $M_{t}=170 \mathrm{GeV}, M_{S}=1 \mathrm{TeV}$ and $X_{t}=0$. Thick lines correspond to $\cos ^{2} 2 \beta=1$ and thin lines to $\cos ^{2} 2 \beta=0$. Solid lines represent $M_{H}$ evaluated in the two-loop approximation and dashed lines in the one-loop approximation. Our chosen value of $\mu\left(t^{*}\right)$ is indicated in the figure with an open diamond and that chosen in ref. [9], $\mu\left(t^{*}\right)=v$, with an open square. We can see that $M_{H}$ evaluated at two-loop is very stable against $\mu\left(t^{*}\right)$ : it varies $\sim 5 \mathrm{GeV}$ in the whole interval $M_{Z} \leq \mu\left(t^{*}\right) \leq M_{S}$ and $\sim 2 \mathrm{GeV}$ between the diamond and the square. However the value of $M_{H}$ evaluated at one-loop is very unstable. In fact the two-loop correction changes from negative at $\mu(t)=M_{Z}(\gtrsim 10 \%)$ to positive at $\mu(t)=M_{S}(\gtrsim 20 \%)$. In the region of our chosen value of $\mu\left(t^{*}\right)$ it is negative and $\sim 3$ $\mathrm{GeV}$ while at $\mu\left(t^{*}\right)=v$ it is positive and greater $\sim 7 \mathrm{GeV}$. Fig. 8 shows that though our choice of $\mu\left(t^{*}\right)$ is more in agreement with perturbation theory than $\mu\left(t^{*}\right)=v$ the difference is however not important when plotting the physical Higgs mass (which was not considered in ref. [9]).

In ref. [10] the diagrammatic and effective potential approaches were used to evaluate the lightest Higgs mass at two-loop order in the framework of the MSSM. Various approximations, like $g=g^{\prime}=0$ in the effective potential, were used, and only the case of zero stop mixing, $X_{t}=0$, was considered. Our results agree with those of ref. 10 within less than $\sim 3 \mathrm{GeV}$. In fact for $M_{t}=150 \mathrm{GeV}, X_{t}=0, \cos ^{2} 2 \beta=1$ and $M_{S}=1 \mathrm{TeV}\left(M_{S}=10 \mathrm{TeV}\right)$ ref. 10 finds $M_{H} \sim 107 \mathrm{GeV}\left(M_{H} \sim 110 \mathrm{GeV}\right)$ while we find from Figs. 6a and 6b $M_{H} \sim 103 \mathrm{GeV}\left(M_{H} \sim 110 \mathrm{GeV}\right)$. We consider this agreement as satisfactory given the approximations used in ref. 10. The fact that two-loop corrections found in ref. [10 are sizeable with respect to the one-loop result can be explained as a consequence of the approximation used there to estimate the oneloop result. Taking $M_{S}=10 \mathrm{TeV}$ and $M_{t}=150 \mathrm{GeV}$, they found $m_{H}=138 \mathrm{GeV}$ at one-loop using a simple approximation that takes into account only the leading part $(\sim$ $\left.M_{t}^{4} \log \left(M_{S}^{2} / M_{t}^{2}\right)\right)$ of the corrections, as it is common practice in some phenomenological analysis. Actually, a slightly more sofisticated approximation (as the one labelled $1 \beta$ in their paper) or the one-loop result obtained by a numerical integration of the RGE (as 
in our approach) gives $m_{H} \sim 115 \mathrm{GeV}$ (both methods agree up to a difference $\sim 2-3$ $\mathrm{GeV}$ due to subleading effects, such as those provided by considering gauge couplings in the effective potential) for the above mentioned values of the parameters. This has to be compared with the two-loop result $M_{H} \sim 110 \mathrm{GeV}$ and shows that the net effect of two-loop corrections is indeed negative and small].

Finally bounds on the lightest Higgs mass have been recently analyzed in ref. [18] in the context of models with Yukawa unification. The results of ref. [18] are presented in the one-loop approximation. They choose $M_{Z}$ as the minimization scale and find larger bounds than in previous estimates. For instance it is found, for $M_{t}=170$ $\mathrm{GeV}$ and any value of the supersymmetric parameters such that $M_{S}<1 \mathrm{TeV}$, that $M_{H} \lesssim 102 \mathrm{GeV}$. We have tried to reproduce their results using our formalism. In fact, their solution for small $\tan \beta$ is close to the fixed point solution where $\tan \beta$ and $m_{t}$ are related through [19]

$$
m_{t}=(196 \mathrm{GeV})\left[1+2\left(\alpha_{3}\left(M_{Z}\right)-0.12\right)\right] \sin \beta .
$$

Now, fixing $M_{S}=1 \mathrm{TeV}$ and maximal mixing $X_{t}^{2}=6 M_{S}^{2}$ we find, for $M_{t}=170 \mathrm{GeV}$ that $\tan \beta=1.74$ from (5.1) and $M_{H} \sim 99 \mathrm{GeV}$ if we fix $\mu\left(t^{*}\right)=M_{Z}$, in agreement with the result of ref. [18]. However this large value of $M_{H}$ is a clear consequence of the chosen minimization scale where two-loop corrections are very large. In fact for $\mu\left(t^{*}\right)=M_{Z}$ our two-loop result gives $M_{H} \sim 85 \mathrm{GeV}$. Moving to the region where perturbation theory is more reliable, as we have done along this paper, we would obtain the final two-loop result, $M_{H} \sim 86 \mathrm{GeV}$. We have plotted in Fig. $9 M_{H}$ as a function of $M_{t}$ for $M_{S}=1 \mathrm{TeV}$ and $\tan \beta$, determined from (5.1), corresponding to the fixed point solution. The solid (dashed) line corresponds to the case of maximal mixing, $X_{t}^{2}=6 M_{S}^{2}$ (zero mixing, $X_{t}=0$ ).

\section{Conclusions}

We have computed in this paper the upper bounds on the mass of the lightest Higgs boson in the MSSM including leading and next-to-leading logarithm radiative corrections. We have used an RG approach by means of a careful treatment of the effective potential in the SM, assuming that supersymmetry is decoupled from the SM, which we have shown to be an excellent approximation for $M_{S} \gtrsim 1 \mathrm{TeV}$ or even much less. Our results have covered the whole parameter space of supersymmetric parameters; in

\footnotetext{
${ }^{6}$ Notice that the results of ref. [10] only contain one- and two-loop leading and next-to-leading contributions to the Higgs mass while, as we have noticed before, our calculation of the Higgs mass includes leading and next-to-leading contributions to all-loop. This effect can be important for the case of large logarithms. We thank R. Hempfling for a discussion on this point.

${ }^{7}$ An interesting result that can be confirmed from ref. [18] (see Fig. 10 in that paper) is that the maximal threshold limit is compatible with the non-existence of color breaking minima, as we already noted. For that reason removing the color breaking minima of the distribution plotted in Fig. 7 does not change the upper bound on the Higgs mass.
} 
fact, when we refer to the MSSM, we simply mean the supersymmetric version of the SM with minimal particle content. We have also included QCD radiative corrections to the top quark mass, which gives a negative contribution to the Higgs mass as a function of the physical (pole) top mass $M_{t}$, and electroweak radiative corrections to the Higgs mass $M_{H}, \Pi\left(M_{H}^{2}\right)-\Pi(0)$, providing a positive contribution to $M_{H}$ and partially cancelling the former ones. Therefore the balance of including the contribution of QCD and electroweak self-energies to the quark and Higgs masses is negative and small $\left(\lesssim 2 \%\right.$ for $\left.M_{t}<200 \mathrm{GeV}\right)$. We also made a reliable estimate of the total uncertainty in the final results, which turns out to be quite small $(\sim 2 \mathrm{GeV})$.

Concerning the numerical results, we have found in particular that for $M_{t} \leq 190$ $\mathrm{GeV}, M_{S} \leq 1 \mathrm{TeV}$ and any value of $\tan \beta$ we have $M_{H}<120 \mathrm{GeV}$ for $X_{t}=0$, and $M_{H}<140 \mathrm{GeV}$ for $X_{t}^{2}=6 M_{S}^{2}$ (maximal threshold effect). We have also applied our calculation to a particularly appealing scenario, namely the assumption that the top has the Yukawa coupling in its infrared fixed point. Then, the bounds are much more stringent. E.g. for $M_{t}=170 \mathrm{GeV}, M_{S} \leq 1 \mathrm{TeV}$ and $X_{t}^{2}=6 M_{S}^{2}$, we find $M_{H} \leq 86$ $\mathrm{GeV}$.

Two papers have recently tried to incorporate radiative corrections to the Higgs mass up to the next-to-leading order and with qualitatively different results. In ref. [9], using the RG approach, positive, and large next-to-leading corrections with respect to the one-loop results were found. In ref. [10], using diagrammatic and effective potential methods in a particular MSSM as well as various approximations, it was found that two-loop corrections are also sizeable, but negative with respect to the one-loop result!

Using the RG approach, as ref. [9], we have found (unlike in ref. [9]) that two-loop corrections are negative with respect to the one-loop result. We have traced back the origin of this disagreement in their choice of the minimization scale. Furthermore the authors of ref. 91 neglected various effects (as the contribution of gauge bosons to the one-loop effective potential, or the wave function renormalization of top quark and Higgs boson) and considered only the case with zero stop mixing.

On the other hand, we have found that the abnormal size of the two-loop corrections obtained in [10] is a consequence of an excesively rough estimate of the one-loop result, but we are in agreement with their final two-loop result. In fact our two-loop results differ from those of ref. [10] by less than 3\%. Also our results show a large sensitivity of the Higgs mass to the stop mixing parameter.

Finally we would like to comment briefly on the generality of our results. As was already stated, we are assuming average squark masses $M_{S}^{2} \gg M_{Z}^{2}$, and that all supersymmetric particle masses are $\gtrsim M_{S}$. If we relax the last assumption, i.e. if some supersymmetric particles were much lighter, the value of the quartic coupling at $M_{S}$ (see eq. (1.3)) would be slightly increased and, correspondingly, our bounds would be slightly relaxed. We have made an estimate of this effect. Assuming an extreme case where all gauginos, higgsinos and sleptons have masses $\sim M_{Z}$, we have found for $M_{S}=1 \mathrm{TeV}$ and $\cos ^{2} 2 \beta=1$ an increase of the Higgs mass $\sim 2 \%$. For values of $\tan \beta$ close to one (as those appearing in infrared fixed point scenarios) the corresponding effect is negligible. On the other hand, our numerical results have been computed 
for $M_{S} \geq 1 \mathrm{TeV}$. For values of $M_{S} \leq 1 \mathrm{TeV}$ the bounds on the lightest Higgs mass are lowered. Hence, in this sense, all our results can be considered as absolute upper bounds.

\section{Acknowledgements}

We thank R. Hempfling and S. Peris for illuminating discussions. We also thank M. Carena, G. Kane, M. Mangano, N. Polonsky, S. Pokorski, A. Santamaría, C. Wagner and F. Zwirner for useful discussions and comments.

\section{Appendix A}

We comment here on the origin and size of threshold contributions to the quartic coupling of the SM, $\lambda$, at the supersymmetric scale. We also evaluate the effective $\mathrm{D}=6$ operators that are relevant for the Higgs potential, showing that for $M_{S} \geq 1 \mathrm{TeV}$ they are negligible.

As it is well known, the one-loop correction to the MSSM effective potential is dominated by the stop contribution

$$
V_{1}^{M S S M}=\frac{3}{32 \pi^{2}}\left\{m_{1}^{4}\left[\log \frac{m_{1}^{2}}{Q^{2}}-\frac{3}{2}\right]+m_{2}^{4}\left[\log \frac{m_{2}^{2}}{Q^{2}}-\frac{3}{2}\right]\right\}
$$

where $m_{1}, m_{2}$ are the two eigenvalues of the stop mass matrix. In a good approximation:

$$
m_{1,2}^{2}=M_{S}^{2}+h_{t}^{2} H_{2} \pm h_{t} H_{2} X_{t}
$$

where $M_{S}^{2}$ is the soft mass of the stops (we are assuming here that this is the same for the left and the right stops, which is a correct approximation since the RGE of both masses are dominated by the same QCD term); $h_{t}$ is the top Yukawa coupling in the MSSM and $X_{t}=A_{t}+\mu \cot \beta$ gives the mixing between stops ( $A_{t}$ is the coefficient of the soft trilinear scalar coupling involving stops and $\mu$ is the one of the usual bilinear Higgs term in the superpotential). Notice also that $M_{S}$ is basically the average of the two stop masses, which is a reasonable choice for the supersymmetric scale when one is dealing with the Higgs potential, as it is our case. Thus we identify the scale $Q$ at which we are evaluating the threshold effects with $M_{S}$.

Now it is straightforward to obtain from (A.1) the contribution to the $H^{4}$ operator (recall that $H$ is the SM Higgs doublet given by eq.(1.2)). That is

$$
\frac{3}{32 \pi^{2}}\left\{\left(\frac{2 X_{t}^{2}}{M_{S}^{2}}\right) h_{t}^{4}-\left(\frac{X_{t}^{4}}{6 M_{S}^{2}}\right) h_{t}^{4}\right\} H^{4},
$$

where have redefined $h_{t}$ to be the usual top Yukawa coupling in the SM. This gives a threshold contribution to the SM quartic coupling

$$
\Delta \lambda=\frac{3 h_{t}^{4}}{16 \pi^{2}} \frac{X_{t}^{2}}{M_{S}^{2}}\left(2-\frac{X_{t}^{2}}{6 M_{S}^{2}}\right)
$$


Alternatively, eq. (A.4) can be obtained diagrammatically from two kinds of diagrams exchanging stops (see the first paper of ref. [4]). Both methods give the same result.

It is worth noticing that A.4 has a maximum for $X_{t}^{2}=6 M_{S}^{2}$, which means that the threshold effect cannot be arbitrarily large. Note also that $X_{t}^{2}=6 M_{S}^{2}$ is barely consistent with the bound from color conserving minimum [13], so the case of maximal threshold represents an extreme situation.

Analogously, we can obtain from (A.1) the relevant effective $\mathrm{D}=6$ operators, i.e. those proportional to $H^{6}$. These turn out to be

$$
\frac{3}{32 \pi^{2}}\left\{\left(\frac{2}{3 M_{S}^{2}}\right)-\left(\frac{X_{t}^{2}}{M_{S}^{4}}\right)+\left(\frac{X_{t}^{4}}{3 M_{S}^{6}}\right)-\left(\frac{X_{t}^{6}}{30 M_{S}^{8}}\right)\right\} h_{t}^{6} H^{6}
$$

which could also be obtained diagrammatically from four kinds of diagrams exchanging stops. It is easy to see that (A.5) produces negligible modifications in the process of electroweak breaking and in the Higgs mass. For example, for $M_{S}=1 \mathrm{TeV}$ and maximal threshold in (A.4), i.e. $X_{t}^{2}=6 M_{S}^{2}$, A.5) gives modifications in the Higgs mass suppressed by a factor $\sim 1 / 150$ with respect to those induced by (A.4). This also means that, regarding the Higgs potential, it is safe to decouple the MSSM from the $\mathrm{SM}$ for $M_{S}=1 \mathrm{TeV}$ or even substantially smaller.

\section{Appendix B}

In this Appendix we shall discuss in more detail the relation between the running mass of the Higgs boson, extracted from the effective potential, and the physical Higgs mass and give the complete expression for the latter.

The physical mass of the Higgs boson field $M_{H}$ is defined as the pole of the propagator and it is both renormalization scheme and gauge (if calculated at all orders of perturbation) independent.

We start with the Lagrangian

$$
\begin{aligned}
\mathcal{L} & =\frac{1}{2}\left(\partial \phi_{0}\right)^{2}-\frac{1}{2} m_{0}^{2} \phi_{0}^{2}+\ldots \\
& =\frac{1}{2}\left(\partial \phi_{R}\right)^{2}-\frac{1}{2} m_{R}^{2} \phi_{R}^{2}+\frac{1}{2} \delta Z_{H}\left(\partial \phi_{R}\right)^{2}-\frac{1}{2} \delta m^{2} \phi_{R}^{2}+\ldots
\end{aligned}
$$

where $\phi_{0}$ and $\phi_{R}=Z_{H}^{-1 / 2} \phi_{0}=\left(1+\delta Z_{H}\right)^{-1 / 2} \phi_{0}$ are the bare and renormalized Higgs fields, and $m_{0}$ and $m_{R}$ are the bare and renormalized masses. With this convention $m_{0}^{2}=m_{R}^{2}+\delta m^{2}-\delta Z_{H} m_{R}^{2}$. Denoting by $\Gamma_{0}\left(\Gamma_{R}\right)$ the inverse of the one-loop corrected bare (renormalized) propagator, we have

$$
\begin{aligned}
\Gamma_{R}\left(p^{2}\right) & =Z_{H} \Gamma_{0}\left(p^{2}\right)=Z_{H}\left[p^{2}-m_{R}^{2}-\delta m^{2}+\delta Z_{H} m_{R}^{2}-\Pi_{0}\left(p^{2}\right)\right] \\
& =p^{2}+\delta Z_{H} p^{2}-\left(m_{R}^{2}+\delta m^{2}\right)-\Pi_{0}\left(p^{2}\right)
\end{aligned}
$$


where $\Pi_{0}\left(p^{2}\right)$ is the (unrenormalized) self-energy of the Higgs boson field and in the last equality we have neglected higher order terms in the perturbative expansion. The renormalized self-energy is defined as

$$
\Pi_{R}\left(p^{2}\right)=\Pi_{0}\left(p^{2}\right)+\delta m^{2}-\delta Z_{H} p^{2} .
$$

Thus

$$
\Gamma_{R}\left(p^{2}\right)=p^{2}-\left(m_{R}^{2}+\Pi_{R}\left(p^{2}\right)\right) .
$$

Consequently, the physical (pole) mass, $M_{H}^{2}$, satisfies the relation

$$
M_{H}^{2}=m_{R}^{2}+\Pi_{R}\left(p^{2}=M_{H}^{2}\right)
$$

On the other hand, the running mass $m_{H}^{2}$, defined as the second derivative of the renormalized effective potential (see eqs. (2.17) and (2.18)), is given by

$$
m_{H}^{2}=\frac{\partial^{2} V_{e f f}}{\partial \phi^{2}}=-\Gamma_{R}\left(p^{2}=0\right)=m_{R}^{2}+\Pi_{R}\left(p^{2}=0\right) .
$$

Comparing (B.5) and (B.6) we have

$$
M_{H}^{2}=m_{H}^{2}+\Delta \Pi
$$

where we have defined (we drop the subscript $\mathrm{R}$ from $\Pi_{R}$ )

$$
\Delta \Pi \equiv \Pi\left(p^{2}=M_{H}^{2}\right)-\Pi\left(p^{2}=0\right) .
$$

Note that $m_{H}^{2}$ defined in $(\mathrm{B} .6)$ is renormalization scheme dependent, as $V_{\text {eff }}$ is, while $M_{H}^{2}$ is not. In particular, in eq.(B.7) both $m_{H}^{2}$ and $\Delta \Pi$ depend on the renormalization scale $\mu$ in such a way that $M_{H}^{2}$ results to be scale independent (at least to $\mathcal{O}(\hbar)$ ). In fact, from eq. (B.3) and the definition

$$
\gamma=\frac{1}{2} \frac{d \log Z_{H}}{d \log \mu}
$$

we easily obtain

$$
\frac{d \Delta \Pi}{d \log \mu}=-2 \gamma M_{H}^{2}
$$

which cancels the scale dependence of $m_{H}^{2}$ (see eq.(4.2)).

We now want to give the complete expression for the physical mass $M_{H}^{2}$. The quantity $\Delta \Pi$ in the Landau gauge is given by the sum of the following terms

$$
\begin{aligned}
\Delta \Pi & =\Delta \Pi_{t t} \quad(\text { top contribution) } \\
& +\Delta \Pi_{W^{ \pm} W^{\mp}}+\Delta \Pi_{Z^{0} Z^{0}} \\
& +\Delta \Pi_{W^{ \pm} \chi^{\mp}}+\Delta \Pi_{Z^{0} \chi_{3}} \quad \text { (gauge and Goldstone bosons contribution) } \\
& +\Delta \Pi_{\chi^{ \pm} \chi^{\mp}}+\Delta \Pi_{\chi_{3} \chi_{3}} \\
& +\Delta \Pi_{H H} \text { (pure scalar bosons contribution). }
\end{aligned}
$$


In eq. B.11) we have taken into account only the contribution from the heaviest fermion, the top, and indicated by $\chi^{ \pm}$and $\chi_{3}$ the charged and the neutral Goldstone bosons, respectively.

The complete expression for the different contributions to $\Delta \Pi$ calculated in the $\overline{M S}$ scheme is, for $M_{H}<2 M_{W}$ :

i) Top contribution:

$$
\Delta \Pi_{t t}=\frac{3 h_{t}^{2}}{8 \pi^{2}}\left\{-2 M_{t}^{2}\left[Z\left(\frac{M_{t}^{2}}{M_{H}^{2}}\right)-2\right]+\frac{1}{2} M_{H}^{2}\left[\log \frac{M_{t}^{2}}{\mu^{2}}+Z\left(\frac{M_{t}^{2}}{M_{H}^{2}}\right)-2\right]\right\} .
$$

ii) Gauge bosons and Goldstone bosons contribution:

$$
\begin{aligned}
& \Delta \Pi_{W^{ \pm} W^{\mp}}+\Delta \prod_{Z^{0} Z^{0}}+\Delta \prod_{W^{ \pm} \chi^{\mp}}+\Delta \Pi_{Z^{0} \chi_{3}} \\
= & \frac{g^{2} M_{W}^{2}}{8 \pi^{2}}\left(I_{W}^{(1)}+I_{W}^{(2)}+\frac{M_{H}^{2}}{2} I_{W}^{(3)}-M_{H}^{2} I_{W}^{(4)}\right. \\
+ & \left.\frac{M_{H}^{2}}{4} I_{W}^{(5)}-\frac{M_{H}^{4}}{2} I_{W}^{(6)}\right)-\frac{1}{2} \frac{g^{2} M_{H}^{2}}{16 \pi^{2}}\left(I_{W}^{(7)}\left(\mu^{2}\right)+I_{W}^{(8)}\left(\mu^{2}\right)\right) \\
+ & \frac{1}{2} \frac{g^{2} M_{H}^{4}}{16 \pi^{2}} I_{W}^{(9)}+\frac{1}{2}\left\{\begin{array}{c}
M_{W} \rightarrow M_{Z} \\
g^{2} \rightarrow g^{2}+g^{\prime 2}
\end{array}\right\},
\end{aligned}
$$

where all the masses in the above expression have to be understood as the physical ones. The $I_{W}^{i}(i=1, \ldots, 9)$ functions read

$$
\begin{aligned}
I_{W}^{1}= & \int_{0}^{1} d x \log \left[1-\frac{M_{H}^{2}}{M_{W}^{2}} x(1-x)-i \epsilon\right]=Z\left(\frac{M_{W}^{2}}{M_{H}^{2}}\right)-2, \\
I_{W}^{2}= & \int_{0}^{1} \int_{0}^{1} d x d y y \log \left[1-\frac{M_{H}^{2}}{M_{W}^{2}} \frac{y(1-y)}{(1-x y)}-i \epsilon\right]=-\frac{11}{12}-\frac{1}{6} \frac{M_{W}^{2}}{M_{H}^{2}} \\
& +\frac{1}{6}\left(4-\frac{M_{H}^{2}}{M_{W}^{2}}\right) Z\left(\frac{M_{W}^{2}}{M_{H}^{2}}\right)+\frac{1}{6} \frac{\left(M_{H}^{2}-M_{W}^{2}\right)^{3}}{M_{H}^{4} M_{W}^{2}} \log \left(1-\frac{M_{H}^{2}}{M_{W}^{2}}\right), \\
I_{W}^{3}= & \int_{0}^{1} \int_{0}^{1} d x d y \frac{y^{3}}{M_{W}^{2}(1-x y)-M_{H}^{2} y(1-y)-i \epsilon}=\frac{5}{6} \frac{1}{M_{H}^{2}}+\frac{1}{3} \frac{M_{W}^{2}}{M_{H}^{4}} \\
& +\frac{1}{3 M_{H}^{2}}\left(\frac{M_{H}^{2}}{M_{W}^{2}}-1\right) Z\left(\frac{M_{W}^{2}}{M_{H}^{2}}\right)+\frac{1}{3 M_{W}^{2}}\left(\frac{M_{W}^{6}}{M_{H}^{6}}-1\right) \log \left(1-\frac{M_{H}^{2}}{M_{W}^{2}}\right), \\
I_{W}^{4}= & \int_{0}^{1} \int_{0}^{1} d x d y \frac{1}{M_{W}^{2}(1-x y)-M_{H}^{2} y(1-y)-i \epsilon}\left(\frac{M_{W}^{4}}{M_{H}^{4}}-1\right) \log \left(1-\frac{M_{H}^{2}}{M_{W}^{2}}\right), \\
= & \left.\frac{1}{2} \frac{1}{M_{H}^{2}}+\frac{1}{2 M_{W}^{2}} Z\left(\frac{M_{W}^{2}}{M_{H}^{2}}\right)+\frac{1}{2 M_{W}^{2}}\right)
\end{aligned}
$$




$$
\begin{aligned}
I_{W}^{5}= & \int_{0}^{1} \int_{0}^{1} \int_{0}^{1} d x d y d z \frac{z(1-z)}{M_{W}^{2}(1-y-z(x-y))-M_{H}^{2} z(1-z)-i \epsilon} \\
= & -\frac{1}{3} \frac{1}{M_{H}^{2}}+\frac{1}{6 M_{W}^{2}}\left(4-\frac{M_{H}^{2}}{M_{W}^{2}}\right) Z\left(\frac{M_{W}^{2}}{M_{H}^{2}}\right) \\
& +\frac{\left(M_{H}^{2}-M_{W}^{2}\right)^{3}}{3 M_{H}^{4} M_{W}^{4}} \log \left(1-\frac{M_{H}^{2}}{M_{W}^{2}}\right)-\frac{1}{6} \frac{M_{H}^{2}}{M_{W}^{4}} \log \frac{M_{H}^{2}}{M_{W}^{2}}+\frac{i \pi}{6} \frac{M_{H}^{2}}{M_{W}^{4}}, \\
I_{W}^{6}= & \int_{0}^{1} \int_{0}^{1} \int_{0}^{1} d x d y d z \frac{z^{3}(1-z)}{\left[M_{W}^{2}(1-y-z(x-y))-M_{H}^{2} z(1-z)-i \epsilon\right]^{2}} \\
= & -\frac{2}{3} \frac{1}{M_{H}^{4}}+\frac{1}{3 M_{W}^{2} M_{H}^{2}}\left(1-\frac{M_{H}^{2}}{M_{W}^{2}}\right) Z\left(\frac{M_{W}^{2}}{M_{H}^{2}}\right) \\
& +\frac{\left(M_{H}^{2}-M_{W}^{2}\right)^{3}}{3 M_{H}^{6} M_{W}^{4}} \log \left(1-\frac{M_{H}^{2}}{M_{W}^{2}}\right)+\frac{1}{3 M_{W}^{4}} \log \left(1-\frac{M_{H}^{2}}{M_{W}^{2}}\right) \\
& -\frac{1}{3} \frac{M_{W}^{2}}{M_{H}^{6}} \log \left(1-\frac{M_{H}^{2}}{M_{W}^{2}}\right)+\frac{1}{3 M_{W}^{4}} \log \frac{M_{W}^{2}}{M_{H}^{2}}+\frac{i \pi}{3} \frac{1}{M_{W}^{4}}, \\
I_{W}^{7}= & \int_{0}^{1} d x(1+2 x) \log \left[\frac{M_{W}^{2} x-M_{H}^{2} x(1-x)-i \epsilon}{\mu^{2}}\right] \\
= & -4+2 \log \frac{M_{W}^{2}}{\mu^{2}}+\frac{M_{W}^{2}}{M_{H}^{2}}+\left(\frac{M_{W}^{4}}{M_{H}^{4}}-3 \frac{M_{W}^{2}}{M_{H}^{2}}+2\right) \log \left(1-\frac{M_{H}^{2}}{M_{W}^{2}}\right), \\
I_{W}^{8}= & \int_{0}^{1} d x \log \left[\frac{M_{W}^{2} x-i \epsilon}{\mu^{2}}\right]=-1+\log \frac{M_{W}^{2}}{\mu^{2}}, \\
I_{W}^{9}= & \int_{0}^{1} \int_{0}^{1} d x d y \frac{1}{M_{W}^{2} x-M_{H}^{2} y-i \epsilon} \\
= & \left(\frac{1}{M_{W}^{2}}-\frac{1}{M_{H}^{2}}\right) \log \left(1-\frac{M_{H}^{2}}{M_{W}^{2}}\right)+\frac{1}{M_{W}^{2}} \log \frac{M_{W}^{2}}{M_{H}^{2}}+\frac{i \pi}{M_{W}^{2}}, \\
z= &
\end{aligned}
$$

where $Z(x)$ is the function

$$
\begin{aligned}
Z(x) & = \begin{cases}2 A \tan ^{-1}(1 / A), & \text { if } x>1 / 4 \\
A \log [(1+A) /(1-A)], & \text { if } x<1 / 4\end{cases} \\
A & \equiv|1-4 x|^{1 / 2} .
\end{aligned}
$$

The terms containing the factor $\log \left(1-M_{H}^{2} / M_{W, Z}^{2}\right)$ develop an imaginary part in the region $M_{W, Z}<M_{H}<2 M_{W}$ corresponding to the unphysical decays $H \rightarrow W^{ \pm} \chi^{\mp}, Z^{0} \chi^{3}$. However, this imaginary part, along with the whole factor $\log \left(1-M_{H}^{2} / M_{W, Z}^{2}\right)$, cancels in (B.13). In fact, using (B.14), (B.13) can be written as:

$$
\begin{aligned}
& \Delta \Pi_{W^{ \pm} W^{\mp}}+\Delta \Pi_{Z^{0} Z^{0}}+\Delta \Pi_{W^{ \pm} \chi^{\mp}}+\Delta \Pi_{Z^{0} \chi_{3}} \\
= & \frac{g^{2} M_{W}^{2}}{8 \pi^{2}}\left[-3+\frac{5}{4} \frac{M_{H}^{2}}{M_{W}^{2}}+\frac{1}{2}\left(3-\frac{M_{H}^{2}}{M_{W}^{2}}+\frac{M_{H}^{4}}{4 M_{W}^{4}}\right) Z\left(\frac{M_{W}^{2}}{M_{H}^{2}}\right)-\frac{M_{H}^{4}}{8 M_{W}^{4}} \log \frac{M_{H}^{2}}{M_{W}^{2}}\right.
\end{aligned}
$$




$$
\left.-\frac{3 M_{H}^{2}}{4 M_{W}^{2}} \log \frac{M_{W}^{2}}{\mu^{2}}+\frac{i \pi}{8} \frac{M_{H}^{2}}{M_{W}^{2}}\right]+\frac{1}{2}\left\{\begin{array}{c}
M_{W} \rightarrow M_{Z} \\
g^{2} \rightarrow g^{2}+g^{\prime 2}
\end{array}\right\} .
$$

There is also an explicit imaginary part in (B.16) giving rise to

$$
\operatorname{Im}\left(\Delta \Pi_{W^{ \pm} W \mp}+\Delta \Pi_{Z^{0} Z^{0}}+\Delta \Pi_{W^{ \pm} \chi^{\mp}}+\Delta \Pi_{Z^{0} \chi_{3}}\right)=\frac{3 g^{2}}{128 \pi} \frac{M_{H}^{4}}{M_{W}^{2}}
$$

which will also cancel, as we will see.

iii) Pure scalar bosons contribution: the contribution to $\Delta \Pi$ coming from the pure scalar sector deserves more attention and we want to discuss it in more details.

It is well-known that in the Landau gauge the Goldstone bosons $\chi$ 's do have a field dependent mass $m_{\chi}(\phi)=-m^{2}+\lambda \phi^{2} / 2$ which vanishes at the minimum of the potential $V_{\text {eff }}(\phi)$. As a consequence, the running mass $m_{H}$ presents an infrared logarithmic divergence when Goldstone bosons are included in the effective potential $V_{\text {eff }}(\phi)$. On the other hand, the physical mass $M_{H}$ must be finite and gauge independent, so the divergent contribution coming from the Goldstone bosons to $m_{H}^{2}$ must be cancelled by an equal (and opposite in sign) contribution of the same excitations to $\Delta \Pi$. To see it explicitly, one can imagine the Goldstone bosons to have a fictitious mass $m_{\chi}$ and calculate their contribution $\Delta m_{H}^{2}$ to the running square mass $m_{H}^{2}$. It is not difficult to see that this contribution from the effective potential is

$$
\Delta m_{H}^{2}=\frac{3}{128 \pi^{2}} \frac{g^{2} M_{H}^{4}}{M_{W}^{2}}\left[3 \log \frac{M_{H}^{2}}{\mu^{2}\left(t^{\star}\right)}+\log \frac{m_{\chi}^{2}}{\mu^{2}\left(t^{\star}\right)}\right]+\mathcal{O}\left(\hbar^{2}\right),
$$

where the scale $\mu\left(t^{\star}\right)$ is defined in the text. On the other hand, the contribution to $\Delta \Pi$ from the pure scalar sector reads

$$
\Delta \Pi_{H H}+\Delta \Pi_{\chi^{ \pm} \chi^{\mp}}+\Delta \Pi_{\chi_{3} \chi_{3}}=\frac{3}{128 \pi^{2}} \frac{g^{2} M_{H}^{4}}{M_{W}^{2}}\left[\pi \sqrt{3}-8+Z\left(\frac{m_{\chi}^{2}}{M_{H}^{2}}\right)-i \pi\right],
$$

where the last term comes from the Feynman diagrams involving Goldstone bosons. The explicit imaginary part in (B.19) would correspond to the unphysical decays $H \rightarrow$ $\chi^{ \pm} \chi^{\mp}, \chi^{3} \chi^{3}$ and cancels against eq. (B.17). Expanding now the function $Z\left(m_{\chi}^{2} / M_{H}^{2}\right)$ around $m_{\chi}^{2}=0$ one can easily show that the logarithmic divergence in $\Delta m_{H}^{2}$ disappears and the final result for the pure scalar bosons contribution to $\Delta M_{H}^{2}$ is finite and given by

$$
\Delta M_{H}^{2}=\frac{3}{128 \pi^{2}} \frac{g^{2} M_{H}^{4}}{M_{W}^{2}}\left[\pi \sqrt{3}-8+4 \log \frac{M_{H}^{2}}{\mu^{2}\left(t^{\star}\right)}\right] .
$$

Finally it is worth making a couple of comments. First, we have included the pure scalar bosons sector in $\Delta \Pi$ for the sake of completeness, but now one is no longer allowed to compare the physical mass given in eq. (B.7) with the running mass since the latter (see eq. (B.18)) is not well defined when Goldstone bosons are taken into account. Secondly, there is no scale dependence in the last expression (B.20) (the scale $\mu\left(t^{\star}\right)$ is fixed (see the text)), in agreement with the fact that the $\lambda$-dependence of the anomalous dimension $\gamma$ of the Higgs field arises at two-loop. 


\section{References}

[1] Z. Kunszt and W.J. Stirling, Phys. Lett. B262 (1991) 54.

[2] Y. Okada, M. Yamaguchi and T. Yanagida, Prog. Theor. Phys. 85 (1991) 1;

J. Ellis, G. Ridolfi and F. Zwirner, Phys. Lett. B257 (1991) 83 and B262 (1991) 477;

R. Barbieri and M. Frigeni, Phys. Lett. B258 (1991) 395;

D. Pierce, A. Papadopoulos and S. Johnson, Phys. Rev. Lett. 68 (1992) 3678.

[3] H. E. Haber and R. Hempfling, Phys. Rev. Lett. 66 (1991) 1815;

A. Yamada, Phys. Lett. B263 (1991) 233;

A. Brignole, Phys. Lett. B281 (1992) 284;

P. H. Chankowski, S. Pokorski and J. Rosiek, Phys. Lett. B274 (1992) 191.

[4] Y. Okada, M. Yamaguchi and T. Yanagida, Phys. Lett. B262 (1991) 54;

R. Barbieri, M. Frigeni and F. Caravaglios, Phys. Lett. B258 (1991) 167;

H. E. Haber and R. Hempfling, Phys. Rev. D48 (1993) 4280.

[5] J. R. Espinosa and M. Quirós, Phys. Lett. B266 (1991) 389.

[6] B. Kastening, Phys. Lett. B283 (1992) 287.

[7] M. Bando, T. Kugo, N. Maekawa and H. Nakano, Phys. Lett. B301 (1993) 83.

[8] C. Ford, D. R. T. Jones, P. W. Stephenson and M. B. Einhorn, Nucl. Phys. B395 (1993) 17.

[9] J. Kodaira, Y. Yasui and K. Sasaki, Hiroshima preprint HUPD-9316, YNUHEPTh-93-102 (November 1993).

[10] R. Hempfling and A. H. Hoang, Phys. Lett. B331 (1994) 99.

[11] F. Abe et al., CDF Collaboration, preprint FERMILAB-PUB-94/097-E and Phys. Rev. Lett. 73 (1994) 225.

[12] G. t'Hooft and M. Veltman, Nucl. Phys. B61 (1973) 455;

W. A. Bardeen, A. J. Buras, D. W. Duke and T. Muta, Phys. Rev. D18 (1978) 3998.

[13] J. M. Frere, D. R. T. Jones and S. Raby, Nucl. Phys. B222 (1983) 11; C. Kounnas, A.B. Lahanas, D.V. Nanopoulos and M. Quirós, Nucl. Phys. B236 (1984) 438; J.-P. Derendinger and C. A. Savoy, Nucl. Phys. B237 (1984) 307; J. A. Casas, A. Lleyda and C. Muñoz, to appear.

[14] N. Gray, D. J. Bradhurst, W. Grafe and K. Schilcher, Z. Phys. C48 (1990) 673.

[15] S. Peris, Mod. Phys. Lett. 16 (1991) 1505. 
[16] H. Arason et al., Phys. Rev. D46 (1992) 3945.

[17] G. Gamberini, G. Ridolfi and F. Zwirner, Nucl. Phys. B331 (1990) 331; B. de Carlos and J. A. Casas, Phys. Lett. B309 (1993) 320

[18] P. Langacker and N. Polonsky, preprint UPR-0594T (February 1994).

[19] V. Barger, M. S. Berger, P. Ohmann and R. J. N. Phillips, Phys. Lett. B314 (1993) 351; M. Carena and C. Wagner, CERN preprint CERN-TH.7321/94. 


\section{Figure Captions}

Fig. 1 Plot of $\phi_{\min }(t) / \xi(t)$ as a function of $\mu(t)$ for $m_{t}=160 \mathrm{GeV}$ and supersymmetric parameters: $M_{S}=1 \mathrm{TeV}, X_{t}=0, \cos ^{2} 2 \beta=1$. The solid (dashed) curve corresponds to the two loop (one-loop) approximation.

Fig. 2 Plot of $\mu\left(t^{*}\right)$ as a function of $m_{t}$ for $\cos ^{2} 2 \beta=1$ (solid line) and $\cos ^{2} 2 \beta=0$ (dashed line) and values of supersymmetric parameters: a) $M_{S}=1 \mathrm{TeV}, X_{t}=0$; b) $M_{S}=10 \mathrm{TeV}, X_{t}=0$; c) $M_{S}=1 \mathrm{TeV}, X_{t}^{2}=6 M_{S}^{2}$; and d) $M_{S}=10 \mathrm{TeV}$, $X_{t}^{2}=6 M_{S}^{2}$.

Fig. 3 Plot of $m_{H, d e r}(t) / m_{H}(t)$ as a function of $\mu(t)$ for $m_{t}=160 \mathrm{GeV}$ and the supersymmetric parameters: $M_{S}=1 \mathrm{TeV}, X_{t}=0, \cos ^{2} 2 \beta=1$.

Fig. 4 The thin lines correspond to $m_{H}$ as a function of $m_{t}$ in the two-loop (solid) and one-loop (dashed) approximation for supersymmetric parameters: $M_{S}=1$ $\mathrm{TeV}, X_{t}=0, \cos ^{2} 2 \beta=1$. The thick solid line is the plot of $M_{H}$, (4.3), as a function of $M_{t},(4.1)$, in the two-loop approximation and for the same values of the supersymmetric parameters.

Fig. 5 Plot of the physical, $M_{H}$ (solid curve), and running, $m_{H}$ (dashed curve) Higgs mass as a function of the scale $\mu(t)$ for supersymmetric parameters $M_{S}=1 \mathrm{TeV}$, $X_{t}=0, \cos ^{2} 2 \beta=1$.

Fig. 6 Plot of $M_{H}$ as a function of $M_{t}$ for $\cos ^{2} 2 \beta=1$ (solid line) and $\cos ^{2} 2 \beta=0$ (dashed line) and values of supersymmetric parameters: a) $M_{S}=1 \mathrm{TeV}, X_{t}=0$; b) $M_{S}=10 \mathrm{TeV}, X_{t}=0$; c) $M_{S}=1 \mathrm{TeV}, X_{t}^{2}=6 M_{S}^{2}$; and d) $M_{S}=10 \mathrm{TeV}$, $X_{t}^{2}=6 M_{S}^{2}$.

Fig. 7 Plot of $M_{H}$ as a function of $\tan \beta$ for $M_{t}=170 \mathrm{GeV}$ and $M_{S}=1 \mathrm{TeV}$. The solid (dashed) curve corresponds to the $X_{t}^{2}=6 M_{S}^{2}\left(X_{t}=0\right)$ case.

Fig. 8 Plot of $M_{H}$ as a function of $\mu\left(t^{*}\right)$ for $M_{t}=170 \mathrm{GeV}, M_{S}=1 \mathrm{TeV}, X_{t}=0$. Solid (dashed) lines are the two-loop (one-loop) approximation, thick (thin) lines correspond to $\cos ^{2} 2 \beta=1\left(\cos ^{2} 2 \beta=0\right)$.

Fig. 9 Plot of $M_{H}$ as a function of $M_{t}$ for $M_{S}=1 \mathrm{TeV}$ and $\tan \beta$ determined from the fixed point condition (5.1). The solid line corresponds to maximal mixing, $X_{t}^{2}=6 M_{S}^{2}$ and the dashed line to $X_{t}=0$. 


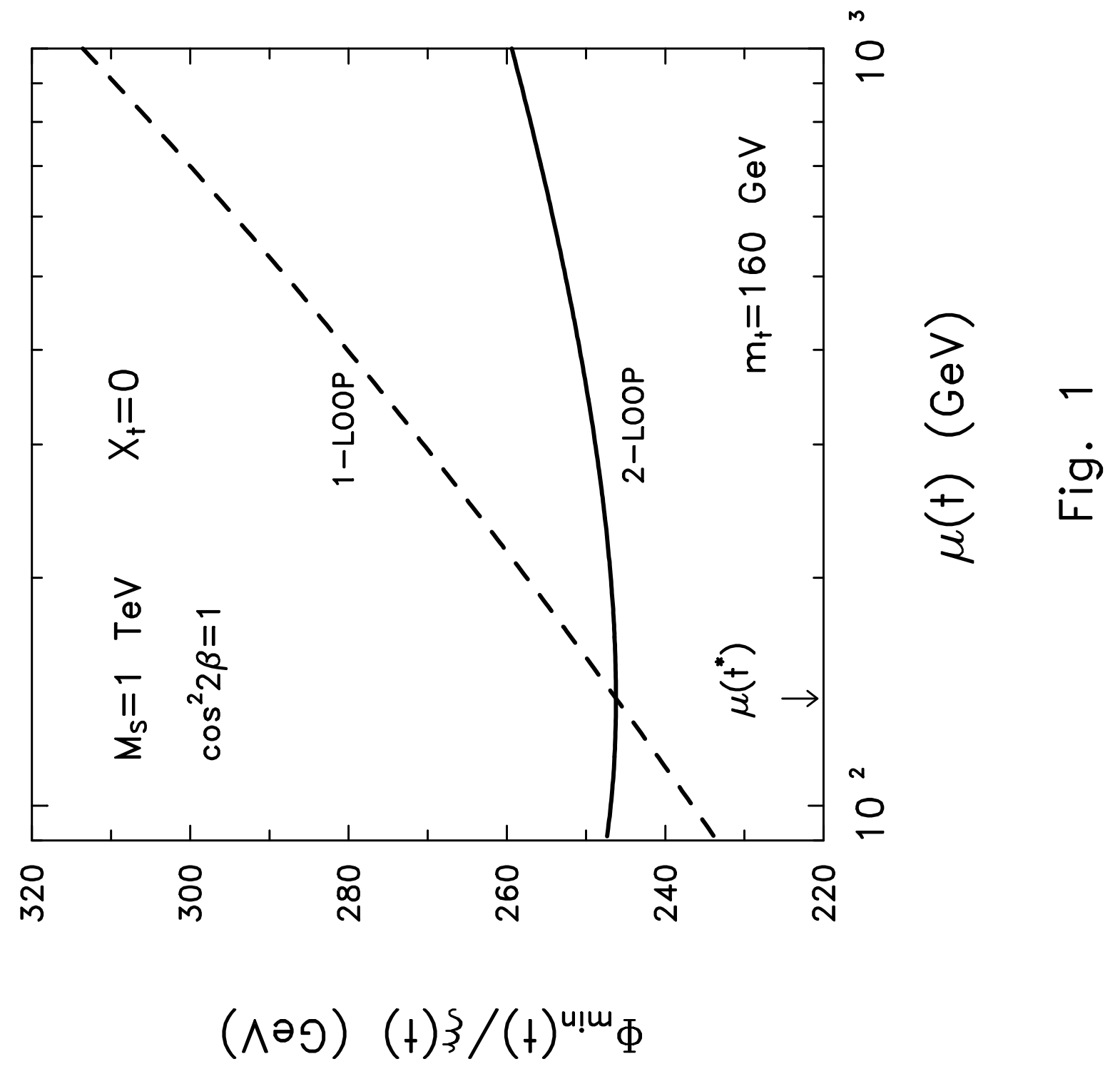


This figure "fig1-1.png" is available in "png" format from: http://arxiv.org/ps/hep-ph/9407389v1 
This figure "fig2-1.png" is available in "png" format from: http://arxiv.org/ps/hep-ph/9407389v1 
This figure "fig3-1.png" is available in "png" format from: http://arxiv.org/ps/hep-ph/9407389v1 


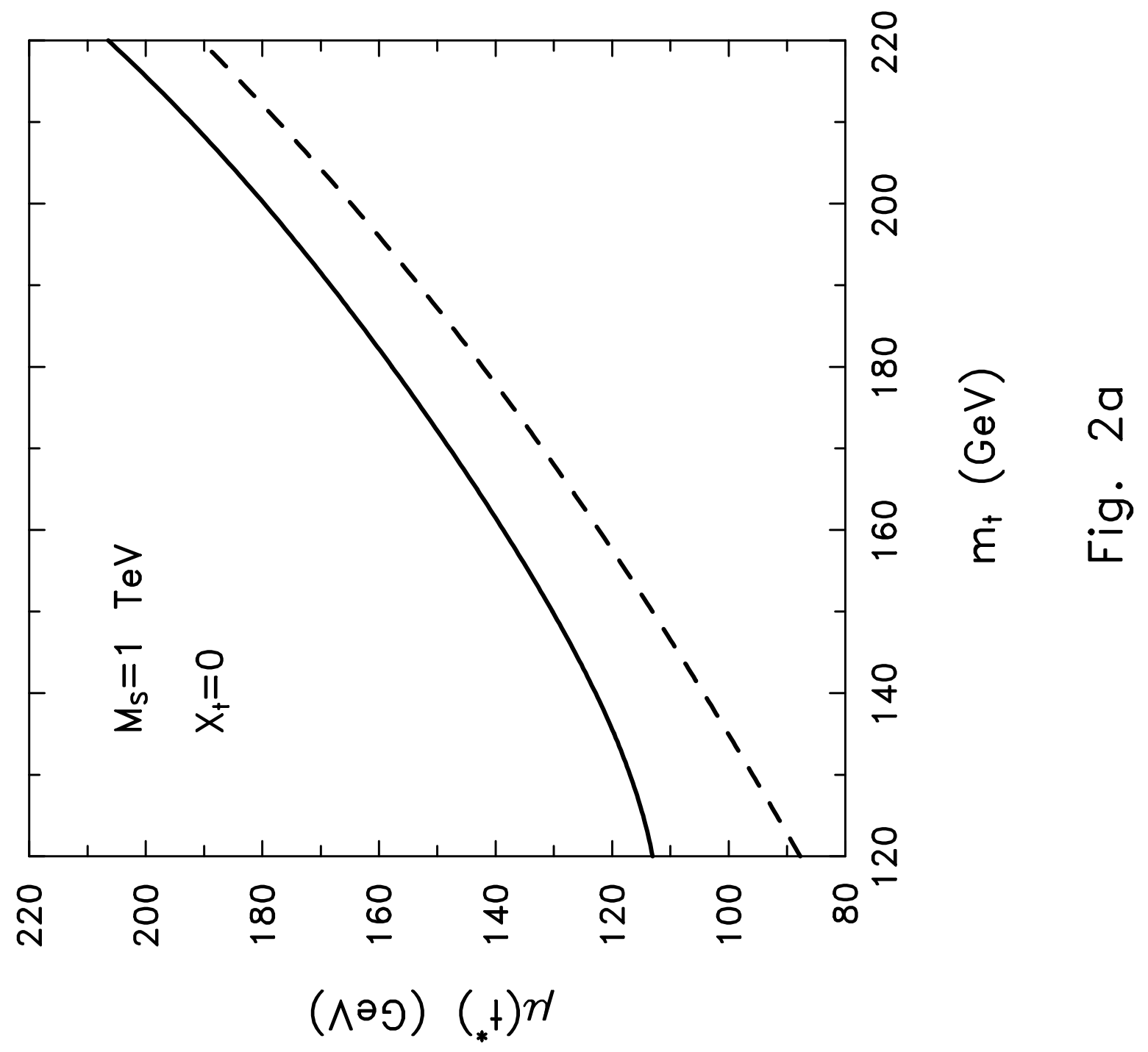




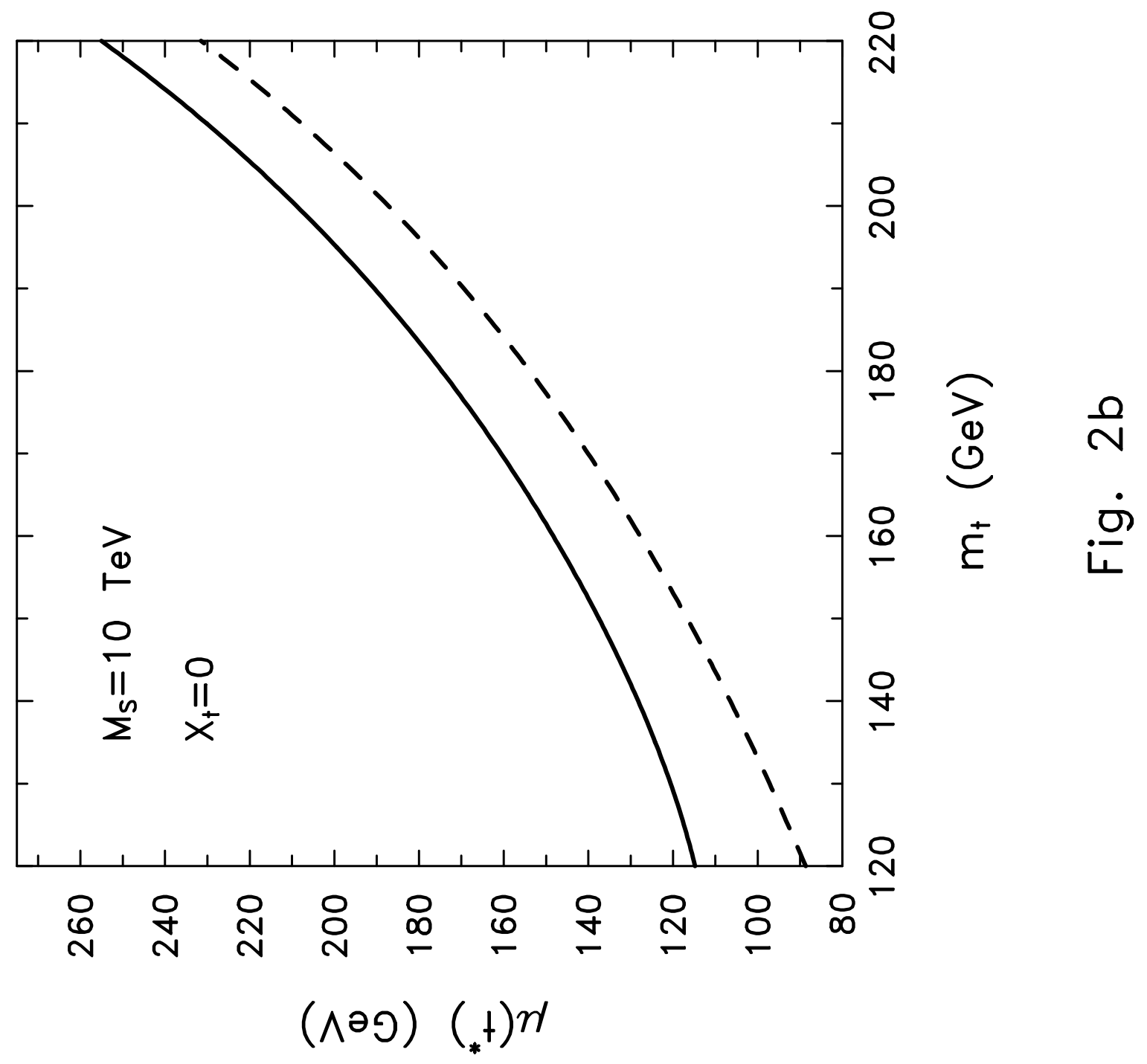




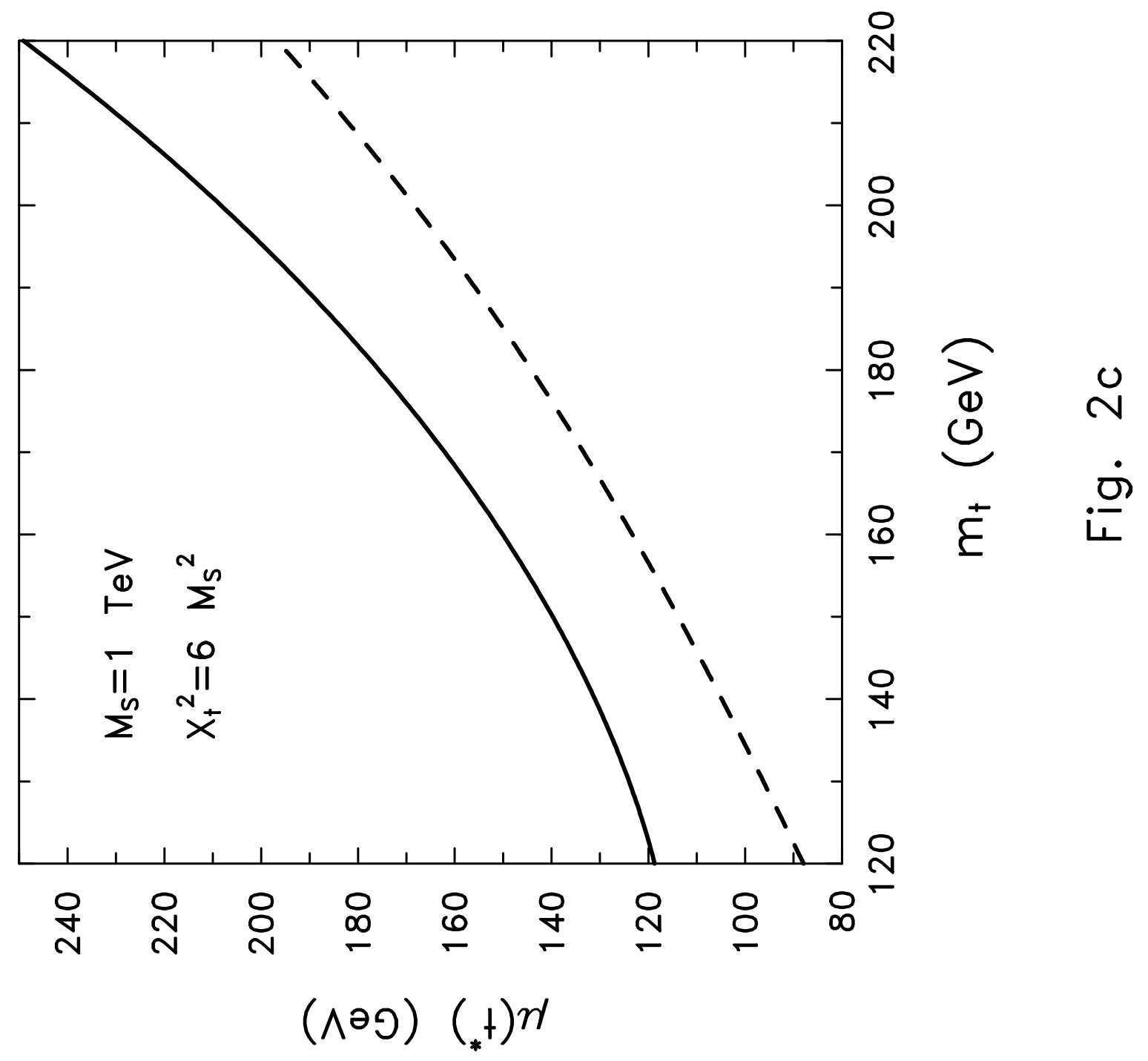




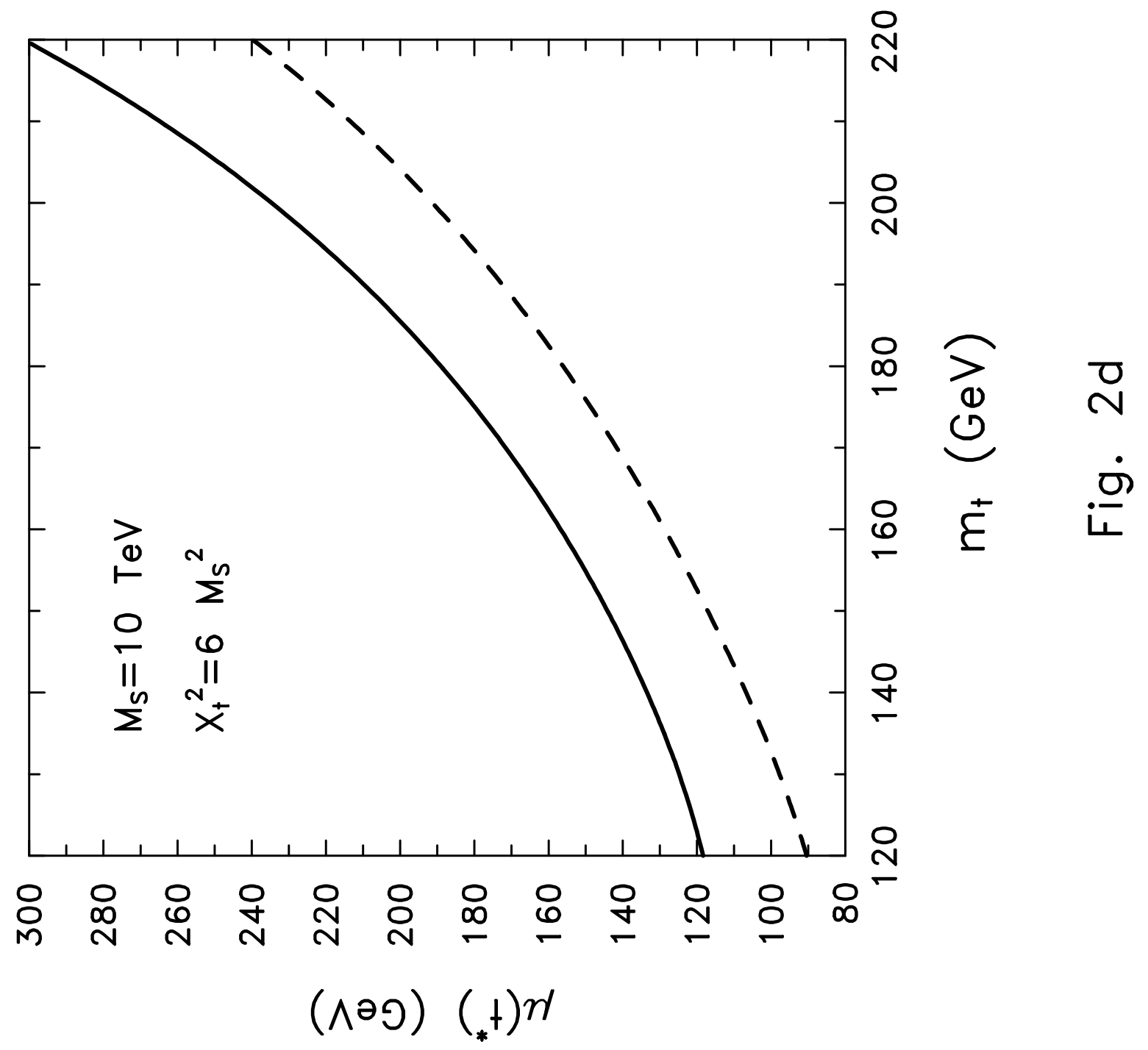


This figure "fig1-2.png" is available in "png" format from: http://arxiv.org/ps/hep-ph/9407389v1 
This figure "fig2-2.png" is available in "png" format from: http://arxiv.org/ps/hep-ph/9407389v1 
This figure "fig3-2.png" is available in "png" format from: http://arxiv.org/ps/hep-ph/9407389v1 


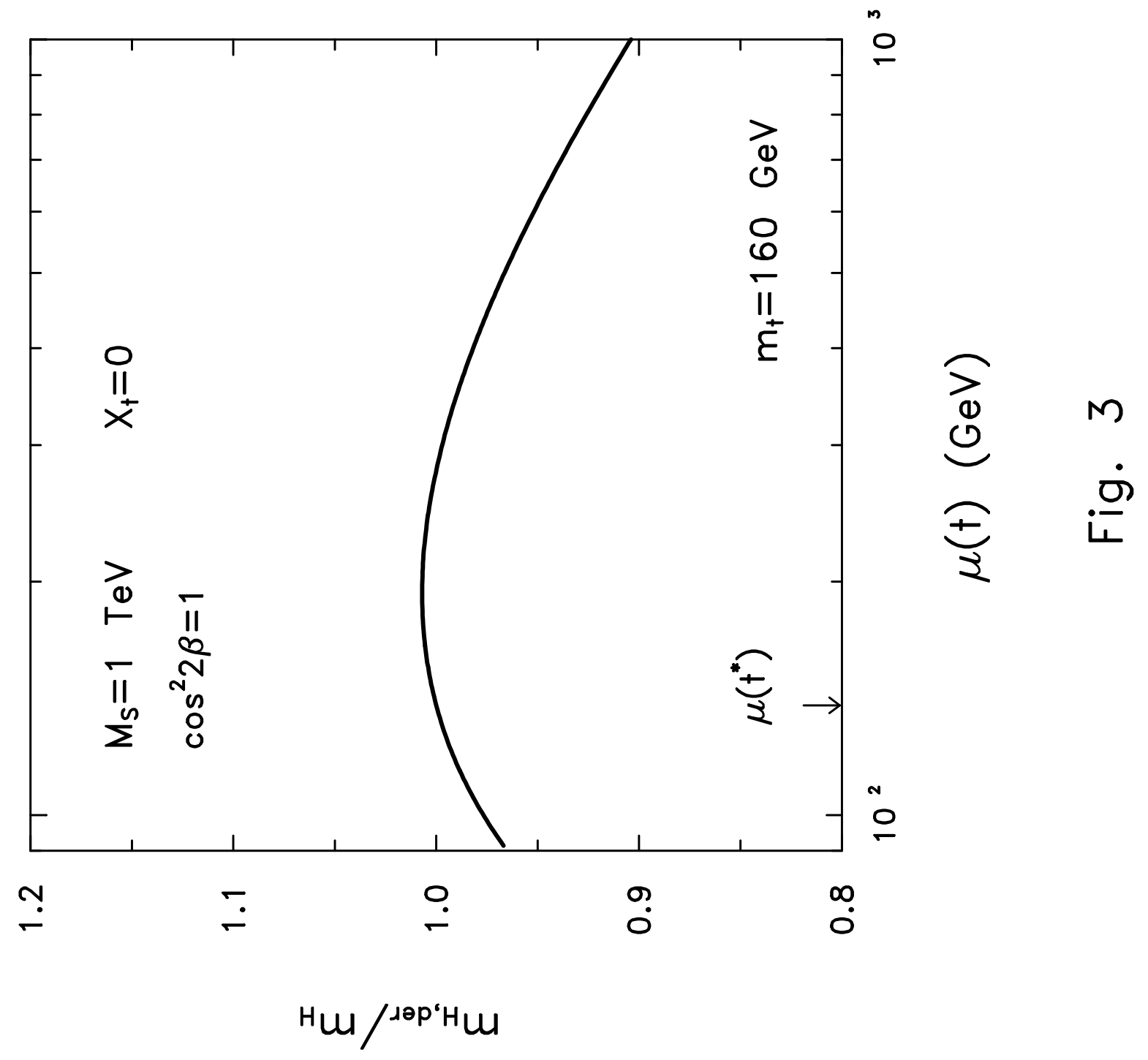


This figure "fig1-3.png" is available in "png" format from: http://arxiv.org/ps/hep-ph/9407389v1 
This figure "fig2-3.png" is available in "png" format from: http://arxiv.org/ps/hep-ph/9407389v1 
This figure "fig3-3.png" is available in "png" format from: http://arxiv.org/ps/hep-ph/9407389v1 


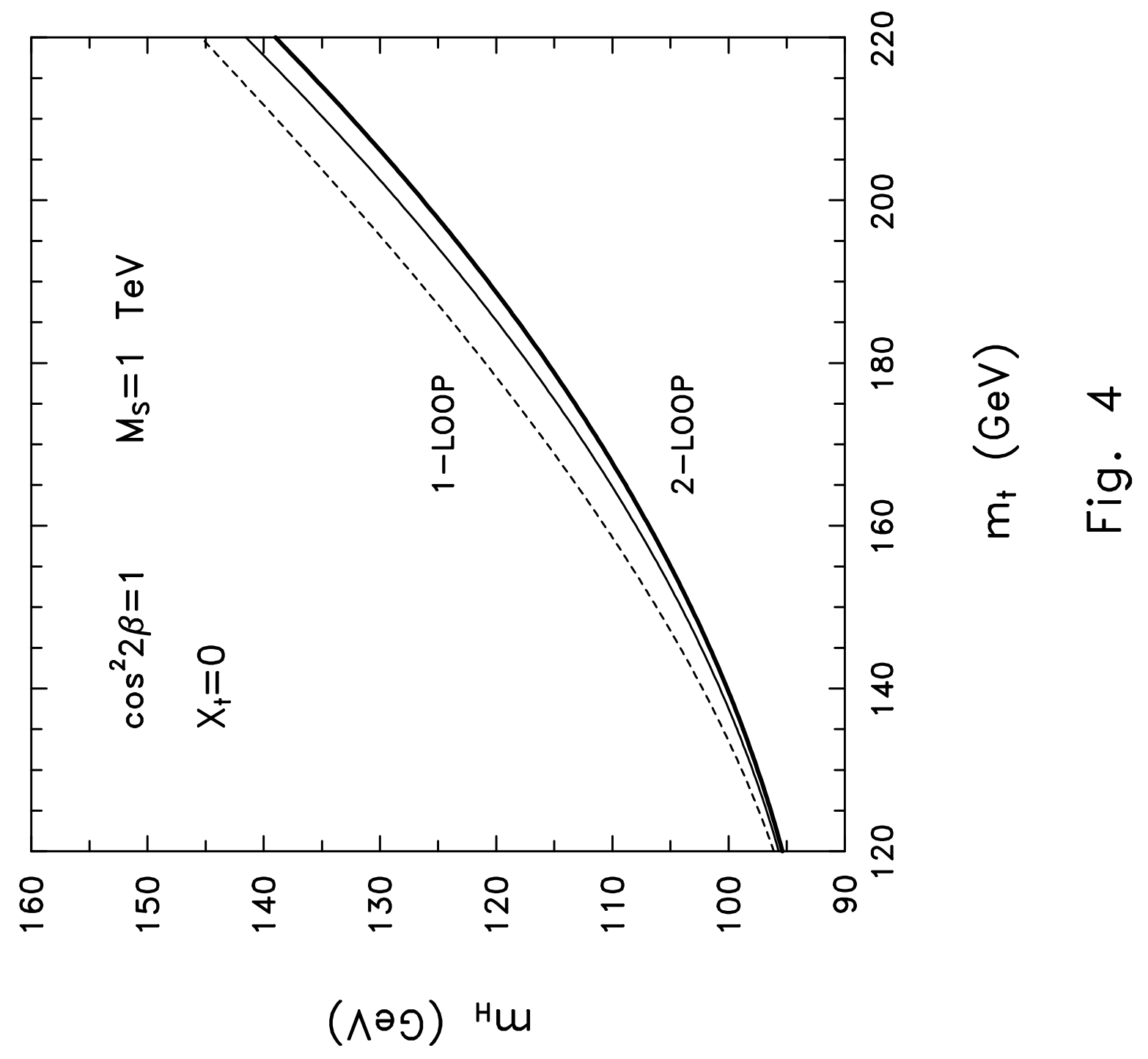


This figure "fig1-4.png" is available in "png" format from: http://arxiv.org/ps/hep-ph/9407389v1 
This figure "fig2-4.png" is available in "png" format from: http://arxiv.org/ps/hep-ph/9407389v1 
This figure "fig3-4.png" is available in "png" format from: http://arxiv.org/ps/hep-ph/9407389v1 


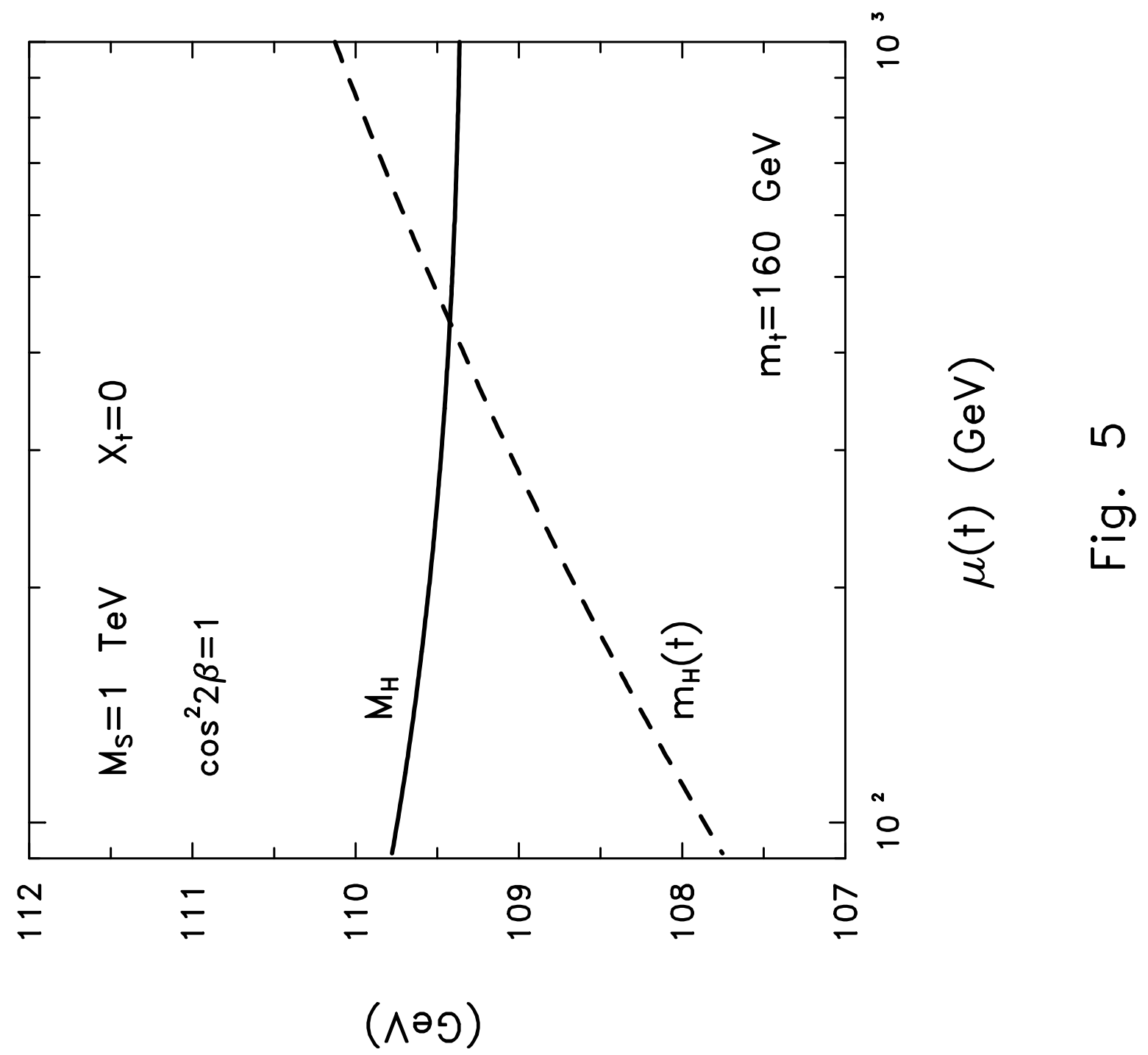


This figure "fig1-5.png" is available in "png" format from: http://arxiv.org/ps/hep-ph/9407389v1 
This figure "fig2-5.png" is available in "png" format from: http://arxiv.org/ps/hep-ph/9407389v1 
This figure "fig3-5.png" is available in "png" format from: http://arxiv.org/ps/hep-ph/9407389v1 


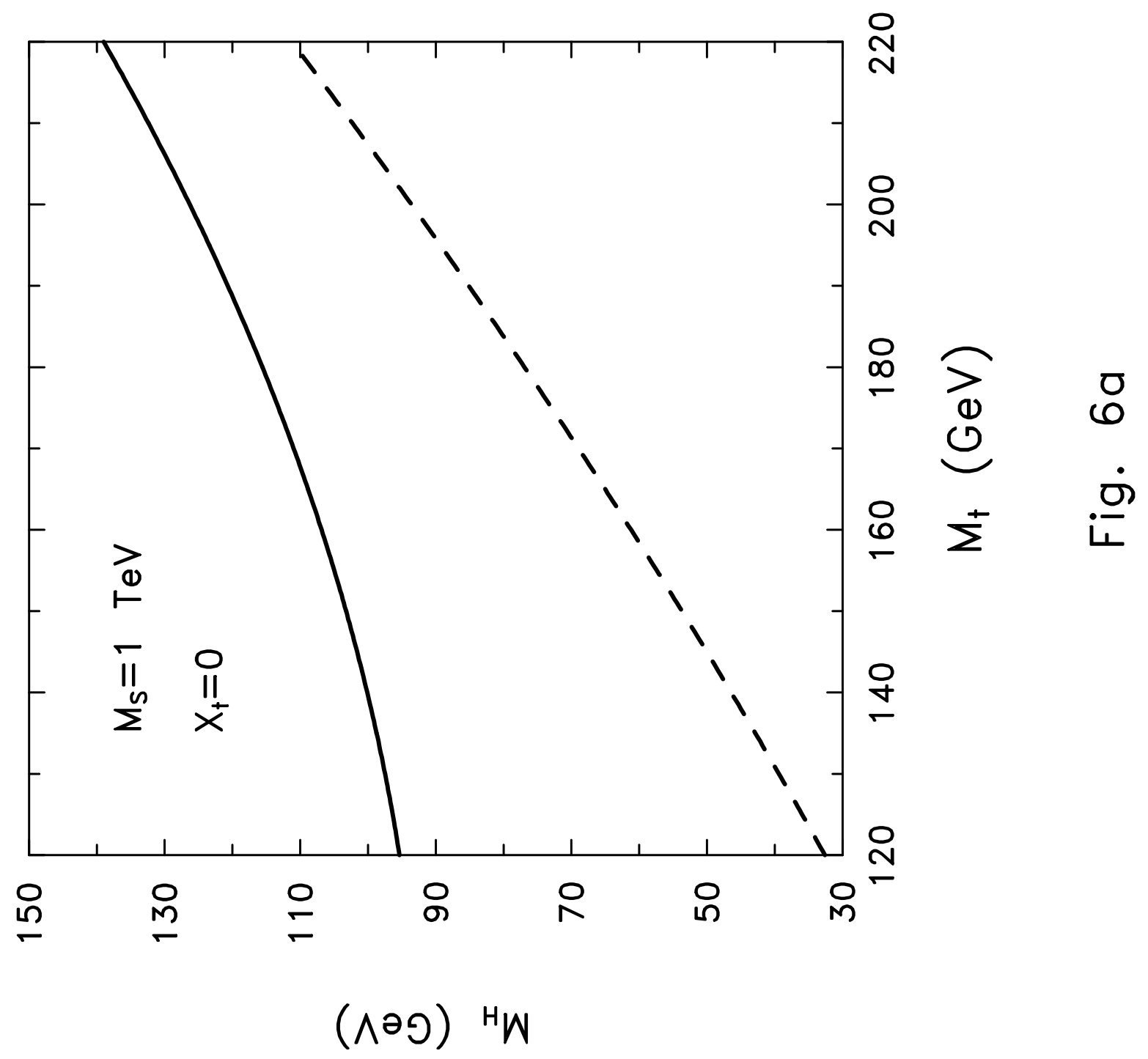




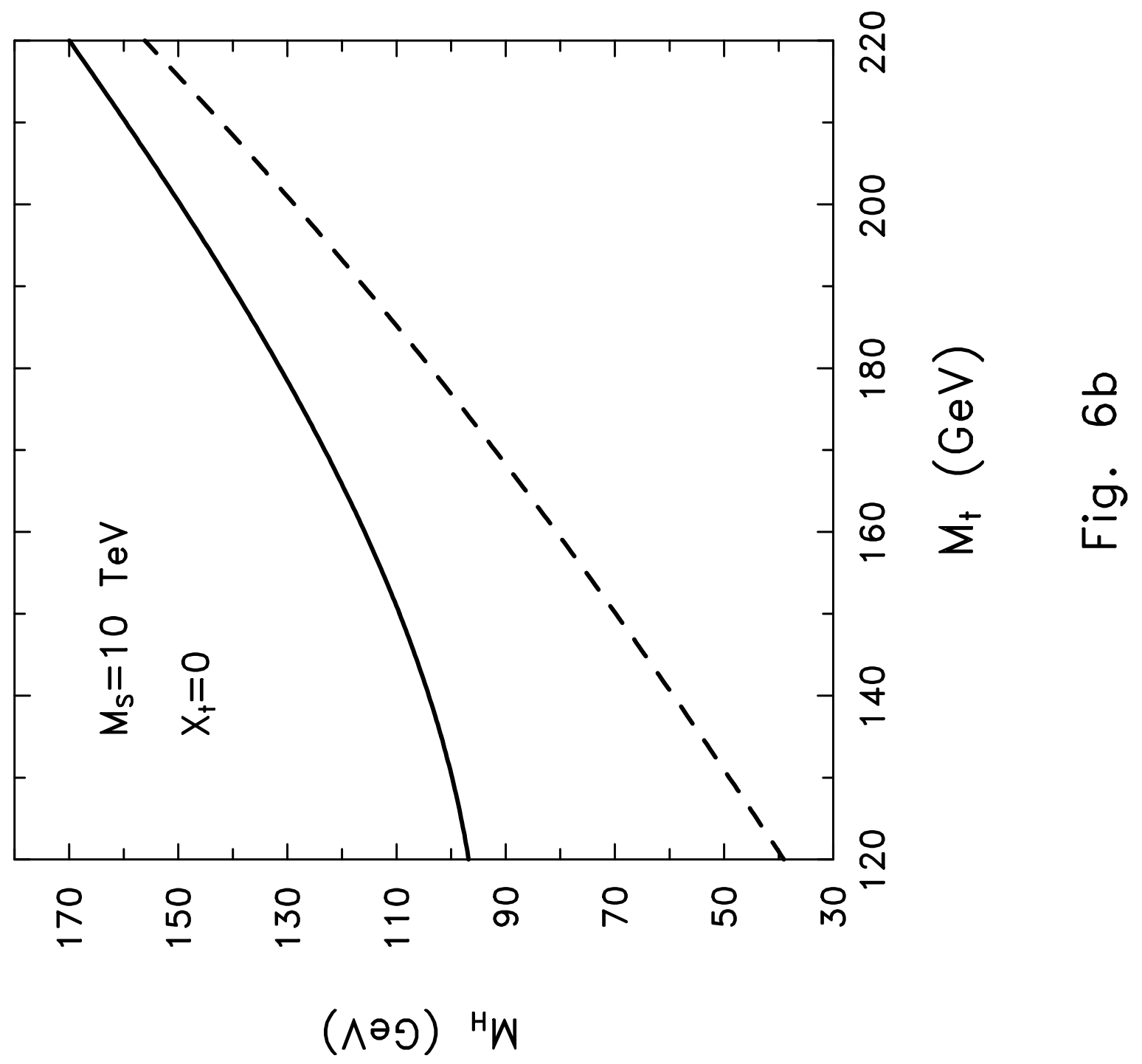




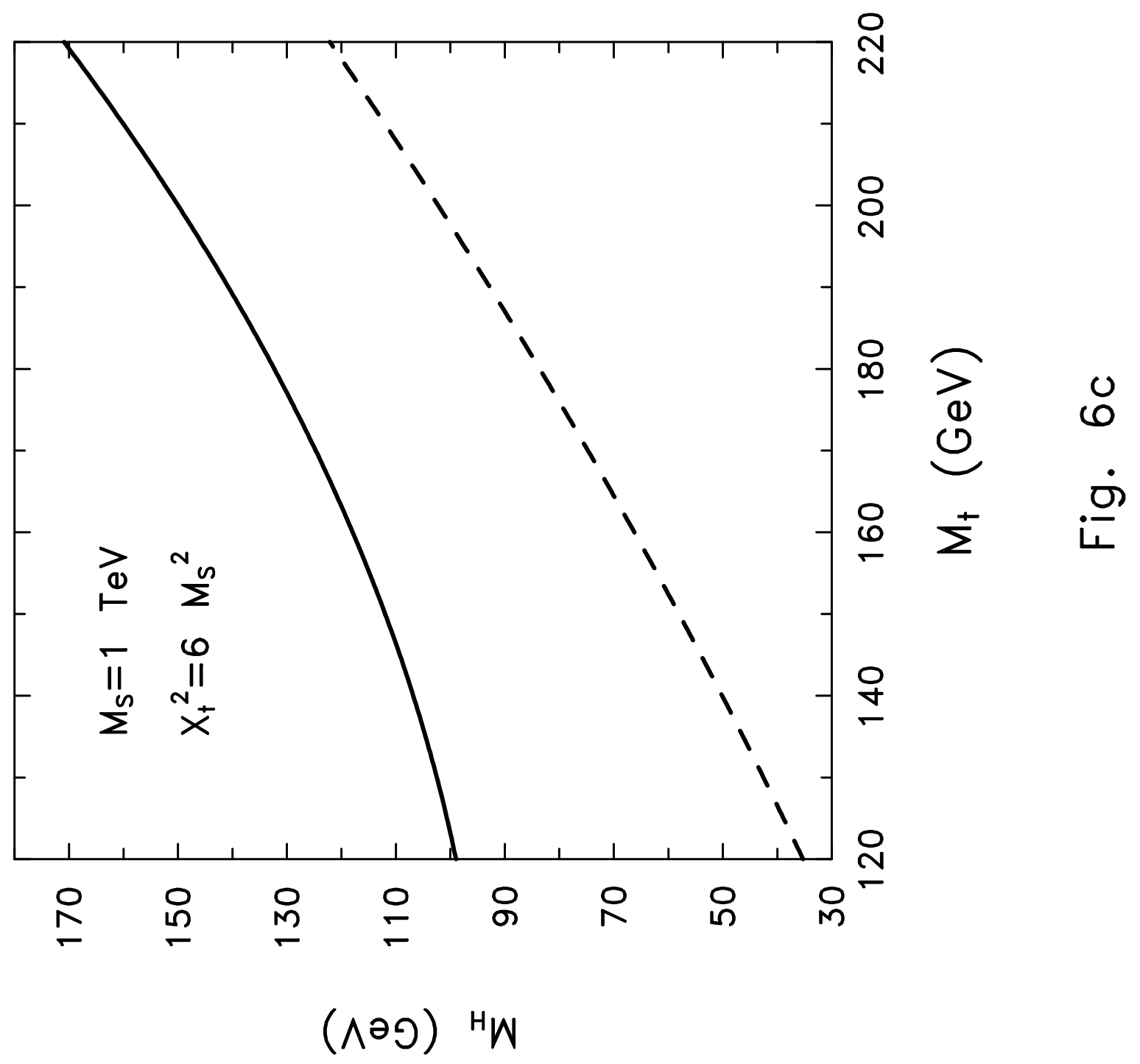




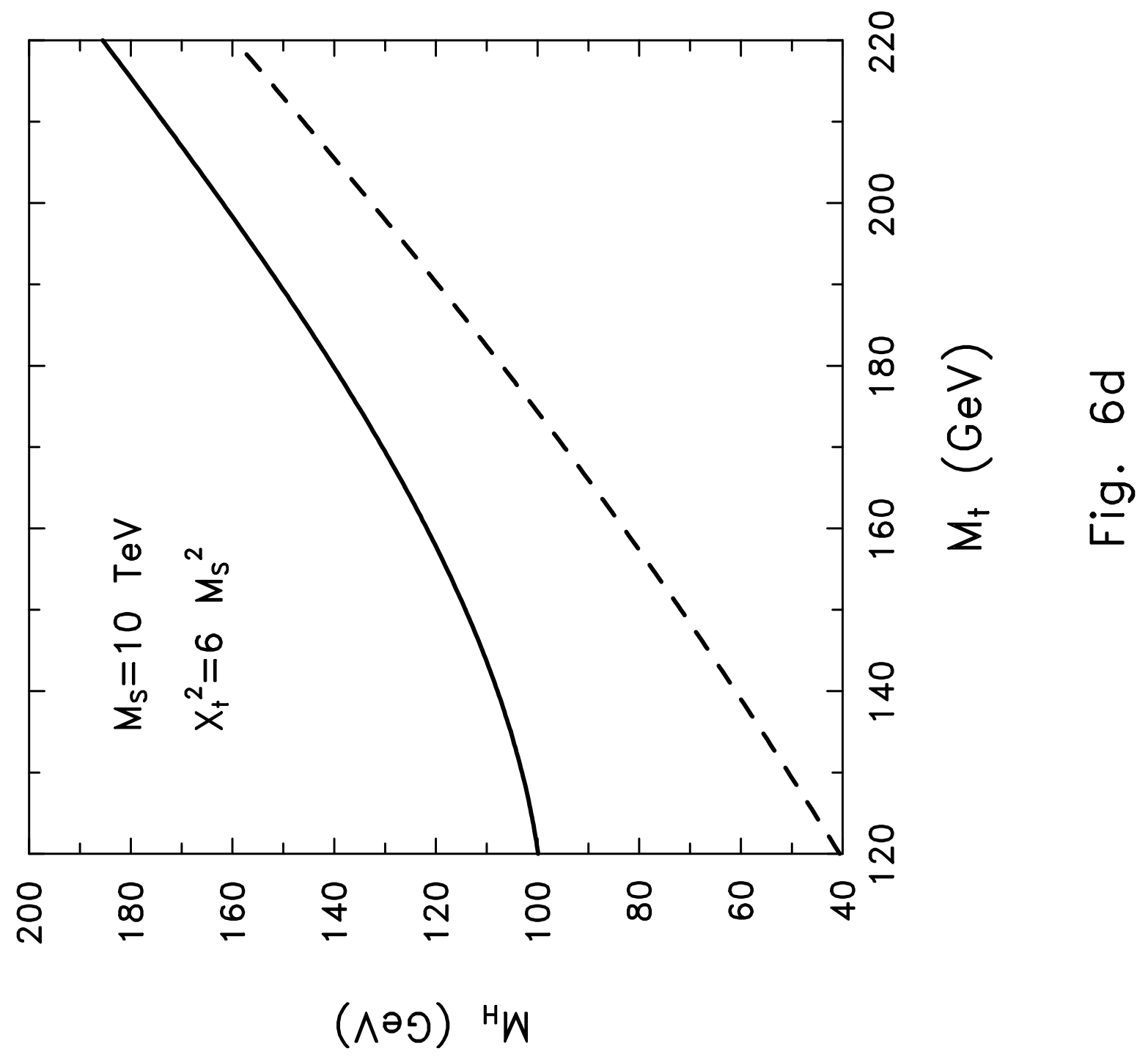




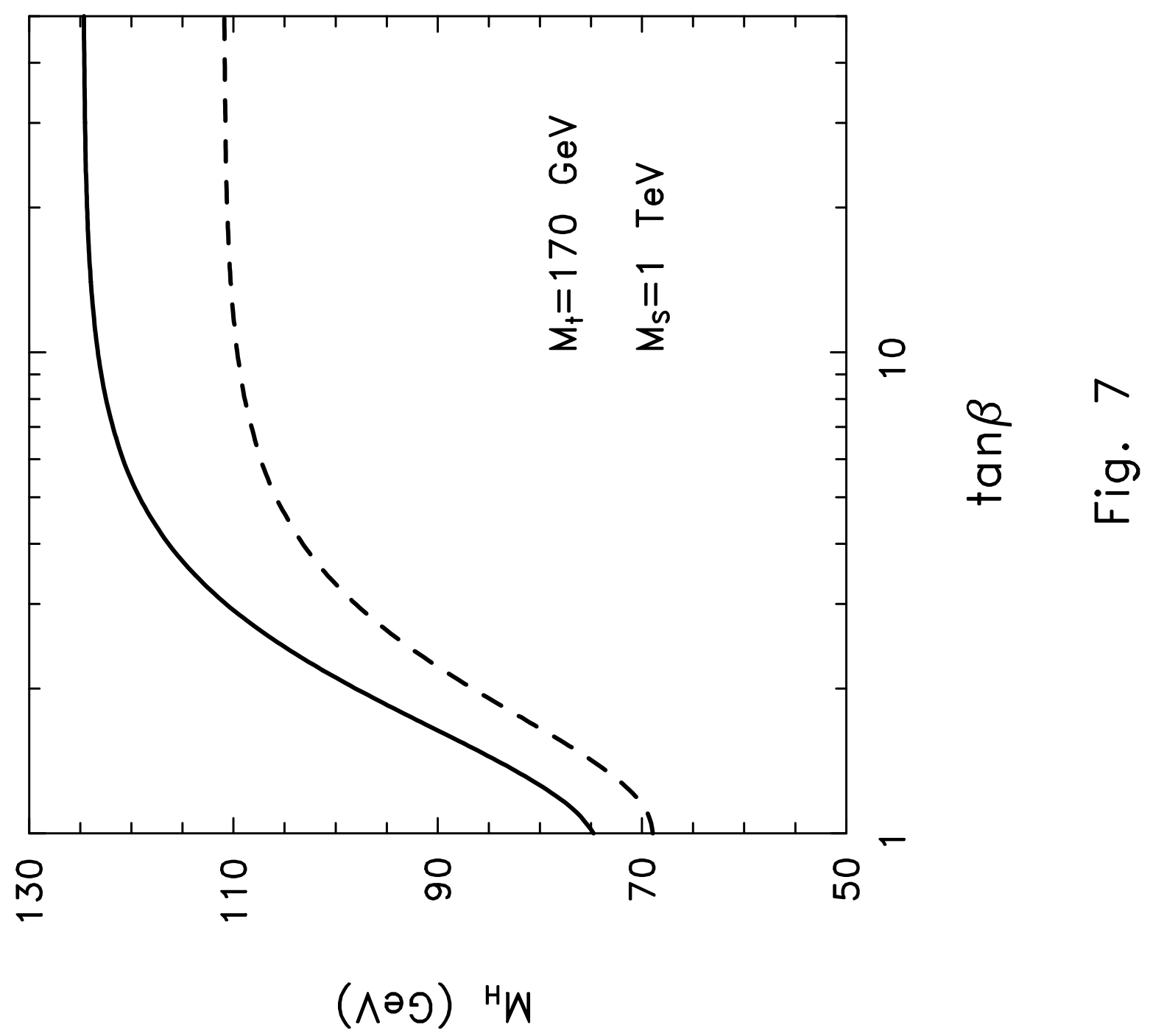




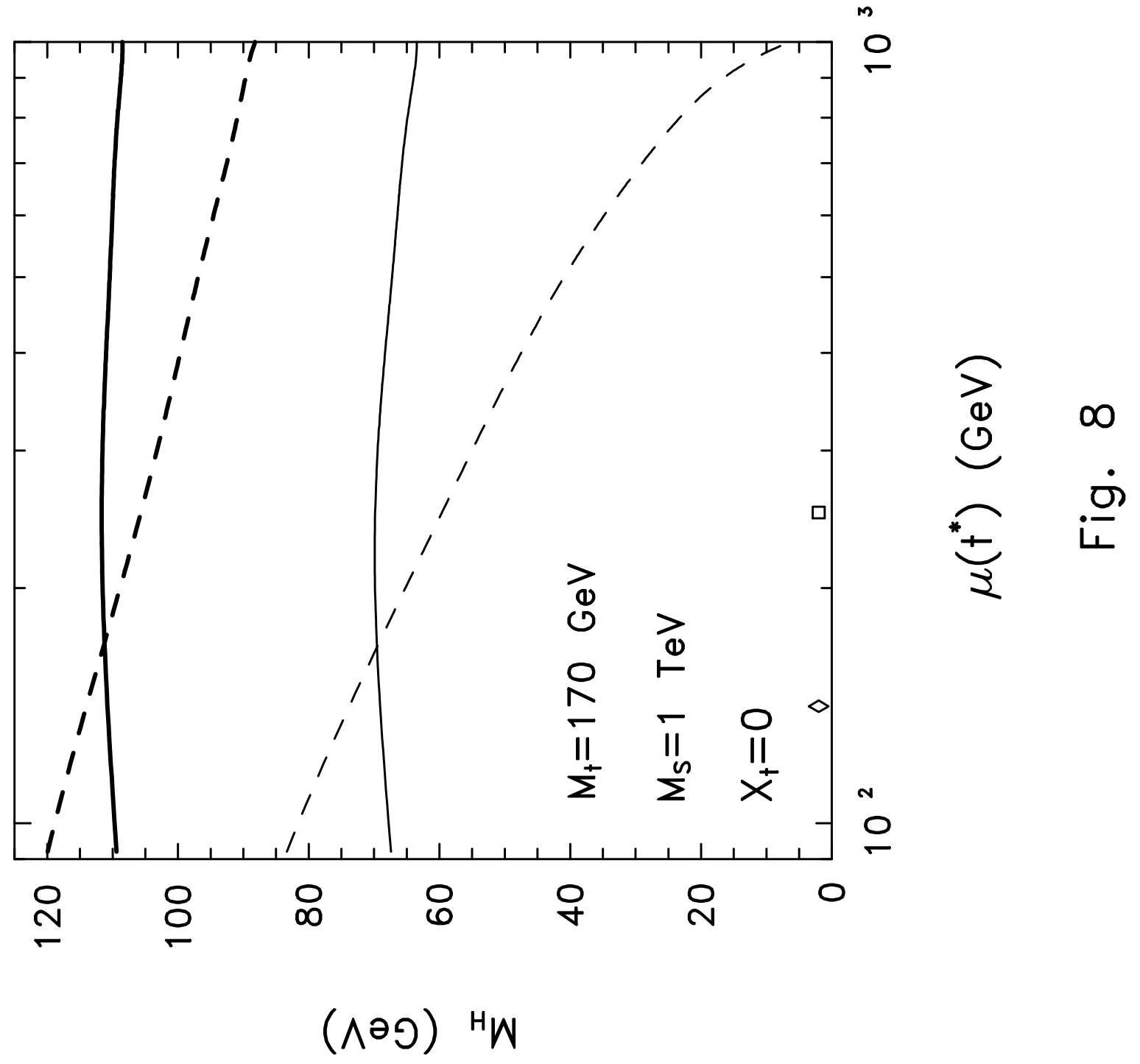




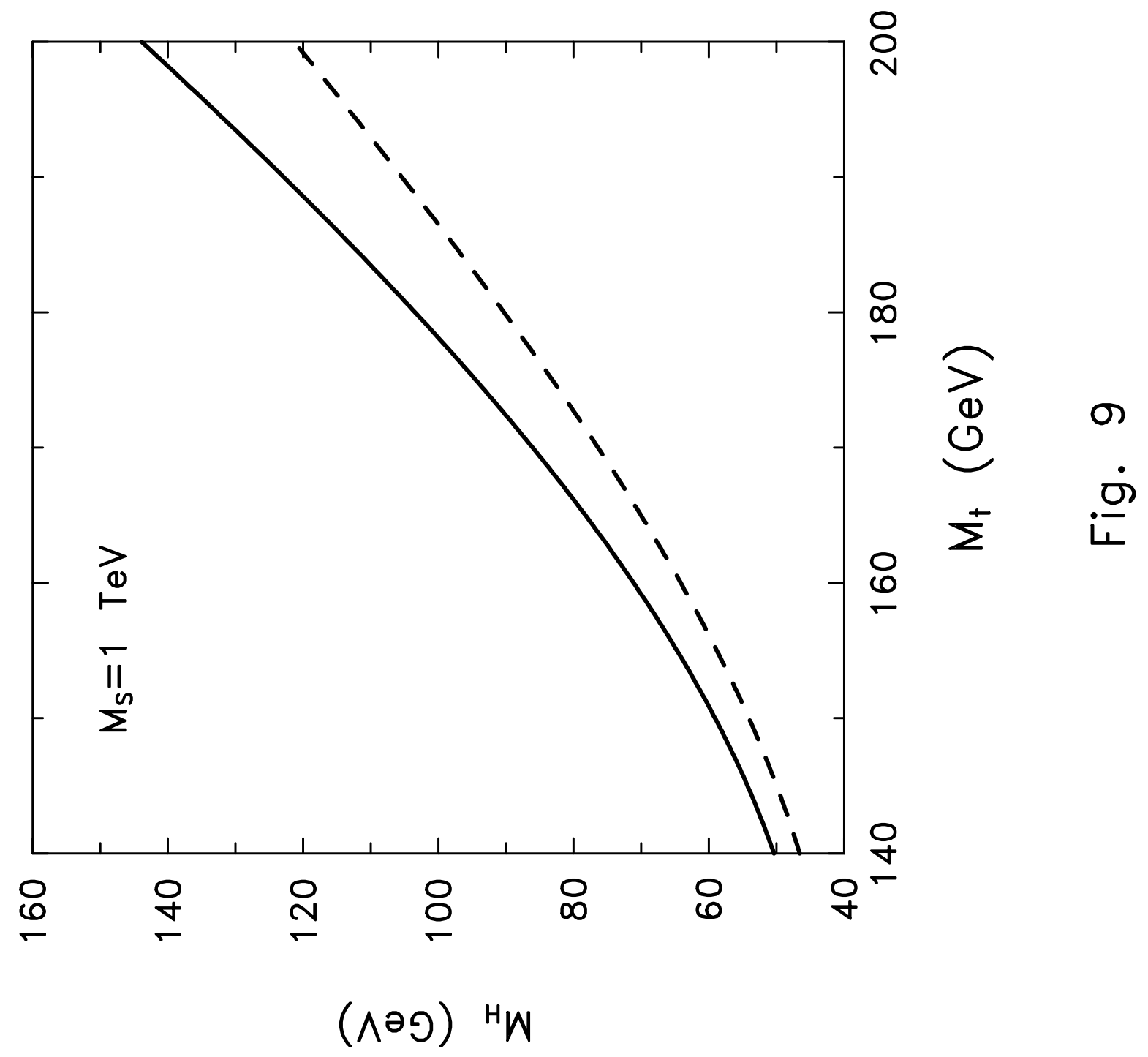

